\title{
Last scene in the large scale rotations of the Western Carpathians as reflected in paleomagnetic constraints
}

\author{
Emö Márton \\ Mining and Geological Survey of Hungary, Palaeomagnetic Laboratory; Columbus 17-23, H-1145 Budapest, Hungary; \\ e-mail: paleo@mbfsz.gov.hu; ORCID ID:0000-0002-2135-8867
}

(C) 2020 Author. This is an open access publication, which can be used, distributed and reproduced in any medium according to the Creative Commons CC-BY 4.0 License requiring that the original work has been properly cited.

Received: 21 November 2019; accepted: 7 August 2020; first published online: 31 August 2020

\begin{abstract}
This paper provides an overview of the paleomagnetic results which constrain the post-Paleogene tectonic development of the Western Carpathians. A group of these results are relevant to the last stage of the Tertiary folding and thrusting of the Silesian, Dukla and Magura nappes of the Outer Western Carpathian and were obtained from Paleogene-Lower Miocene flysch sediments. Both the pre- and post-folding remanences indicate about $50^{\circ} \mathrm{CCW}$ vertical axis rotation with respect to the present orientation. This is about a $60^{\circ}$ rotation relative to stable Europe. It follows that the general orientation of the Silesian and more internal nappes were NW-SE, at least until the mid-Miocene. The CCW vertical axis rotation was co-ordinated with that of the Central Carpathian Paleogene Basin. The termination of the rotation can be estimated from the paleomagnetic data available from the Pieniny andesites which intruded the Pieniny Klippen Belt and the southern part of the Magura Nappe as well as from those obtained for the Neogene intramontane basins which opened up in the Outer and in the Central Western Carpathians. The paleomagnetic vectors for the andesites form two groups. The first group suggests about $45^{\circ} \mathrm{CCW}$ rotation relative to north, while the second shows no rotation. At the present stage of our knowledge it seems likely that some of the andesite bodies were intruded around $18 \mathrm{Ma}$, which is the oldest isotope age for the intrusions of the Wżar Mts, while some other bodies could have been emplaced after the rotation, around $11 \mathrm{Ma}$, which is the youngest isotope age for the Brijarka quarry. Vertical axis CCW rotation was also observed on sediments older than 11.6 Ma in the Orava-Nowy Targ Intramontane Basin which saddles the Magura Nappe and the Central Carpathian Paleogene Basin. However, this rotation was related to fault zone activity and was not attributed to the general rotation of the Outer Western Carpathian nappe system. Paleomagnetic results from the Nowy Sącz Intramontane Basin, which opened over the Magura Nappe, and those for the Central Western Carpathian Turiec Intramontane Basin do not indicate vertical axis rotation. In the first case, the loosely controlled age limit of the termination of the rotation is around $12 \mathrm{Ma}$. Well constrained results from the second basin imply that the rotation was definitely over by $8 \mathrm{Ma}$. Based on the above observations, and aware of the problem of often loose age control on the formation and deformation of the deposits of the intramontane basins, it is tentatively concluded that the large scale CCW rotation of the Central Western Carpathians, together with the Magura, Dukla and Silesian nappes, must have started after $18 \mathrm{Ma}$ and terminated around $11 \mathrm{Ma}$.
\end{abstract}

Keywords: Western Carpathians, large scale rotations, paleomagnetic constraints

\section{INTRODUCTION}

This paper intends to deal with the final stage of the large-scale rotations of the Western Carpathians (Fig. 1) as reflected in the paleomagnetic constraints which are related to the folding and thrusting of the nappe systems of the Outer Western Carpathians over the southern margin of the European Platform and those postdating the large-scale rotations. 


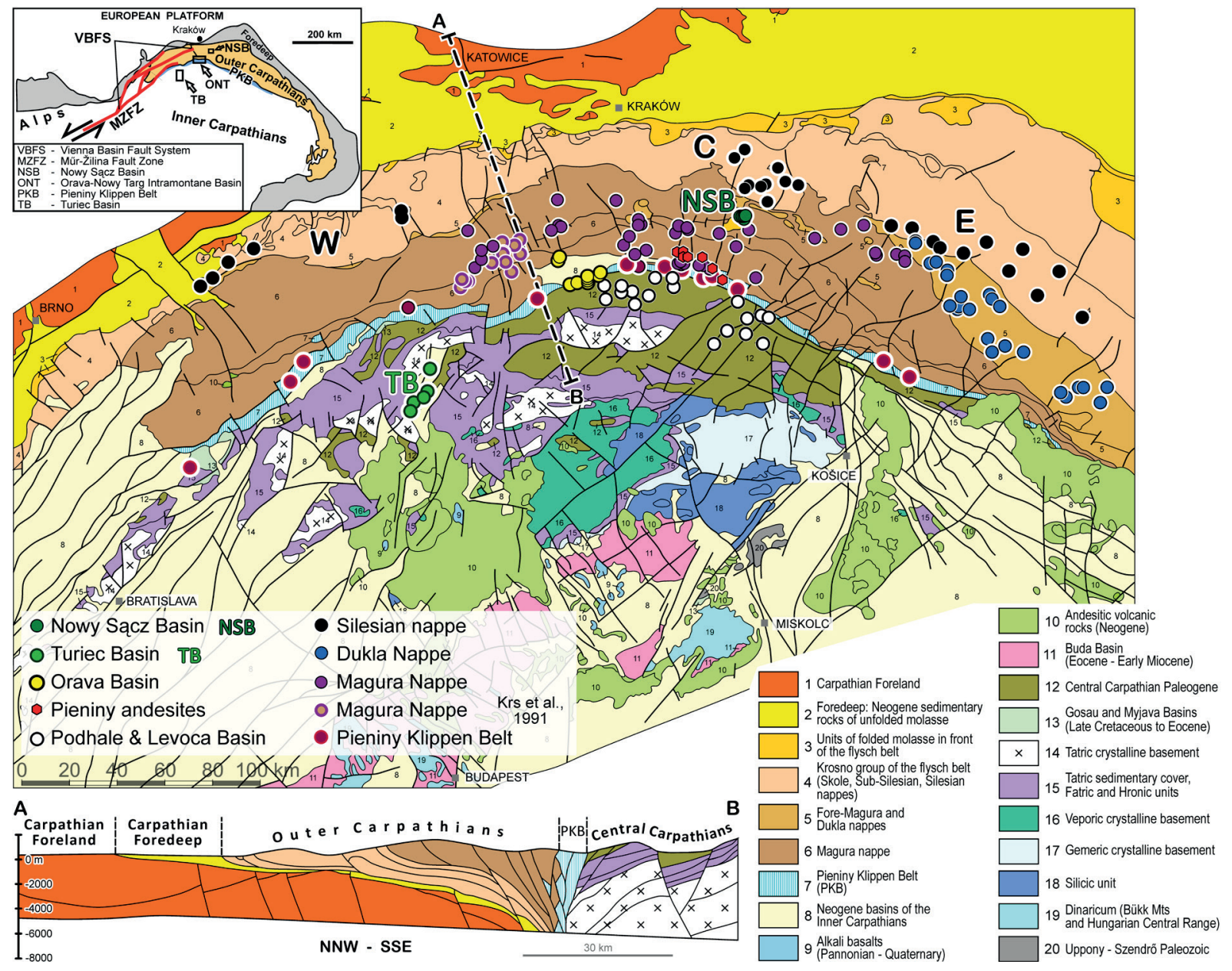

Fig. 1. Structural scheme of the Western Carpathians and adjacent areas (after Lexa et al. 2000, slightly modified) with the distribution of paleomagnetic sampling points in Late Cretaceous and younger rocks. In the upper left corner, a sketch map shows the position of the Mür-Žilina Fault Zone, below the structural map a generalized cross section is drawn from the Carpathian foreland to the Central Carpathians between points A and B on the map

The paleomagnetic results, which serve as the basis for the interpretation, have been published along with the documentation of their quality (Márton et al. 1999, 2004, 2009a, 2009b, 2013, Kiss et al. 2016, Tokarski et al. 2016) or submitted for publication (Turiec Basin, Márton et al. in prep.). Exceptions are those from the Nowy Sącz Intramontane Basin, which is a Neogene basin developed over the Magura Nappe. In this case, the present paper will provide the necessary background information. The basic data for each sedimentary locality/igneous site will be tabulated together with statistical parameters and information about the acquisition of the magnetization in relation to the deformation event responsible for the tilting of the sampled strata (Tab. 1). The tectonic interpretation will be based on the combination of the results from several geographically distributed localities/sites of similar ages available for a particular unit for a certain time (Tab. 2). The reason is that paleomagnetic directions from isolated single localities/sites are only valuable as indications, but not constraints for large scale movements since they may be handicapped by local distorting factors. These factors could be e.g. non-removable partial overprint on the primary magnetization, incomplete tilt correction of the paleomagnetic vector. The latter can cause serious error in paleomagnetic declination when the tilt angle exceeds $30^{\circ}$, and the tilt axis departs from the horizontal at the same time (e.g. the plunging axis of the structure). 


\begin{tabular}{|c|c|c|c|c|c|c|c|c|c|c|c|c|c|c|c|c|}
\hline 㐫 & $\rightarrow$ & - & - & - & - & $\rightarrow$ & - & - & - & - & - & - & N & - & - & - \\
\hline$\stackrel{\circ}{\Xi}$ & $\stackrel{\vartheta}{\sigma}$ & 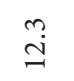 & $\hat{\grave{i}}$ & $\stackrel{\circ}{\circ}$ & $\stackrel{\infty}{\sigma}$ & $\ddot{\sigma}$ & ?్̧ర & $\stackrel{+}{\ddot{n}}$ & $\hat{\sigma}$ & $\stackrel{m}{\sim}$ & $\stackrel{m}{m}$ & $\stackrel{\ddot{g}}{\sim}$ & $\underset{\infty}{\infty}$ & $\hat{b}$ & $\stackrel{\circ}{\circ}$ & $\stackrel{\curvearrowright}{\curvearrowright}$ \\
\hline$\stackrel{\circ}{0}$ & $\vec{i}$ & $\underset{\underset{+}{+}}{\stackrel{g}{ }}$ & $\stackrel{m}{m}$ & $\stackrel{\circ}{\exists}$ & $\stackrel{\curvearrowright}{ \pm}$ & $\stackrel{H}{=}$ & $\stackrel{\circ}{\infty}$ & $\stackrel{H}{\ddot{\theta}}$ & $\hat{\alpha}$ & $\stackrel{\vec{m}}{\dot{m}}$ & $\stackrel{\wedge}{\infty}$ & $\ddot{\Omega}$ & $\stackrel{\infty}{\stackrel{\sim}{-}}$ & $\vec{a}$ & $\stackrel{\infty}{\stackrel{\sim}{-}}$ & ָั \\
\hline $\mid$\begin{tabular}{cc}
0 & 0 \\
\hdashline & 00 \\
0 & 0 \\
0 & 0
\end{tabular} & 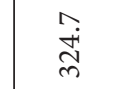 & $\stackrel{m}{m}$ & $\underset{m}{\stackrel{+}{m}}$ & $\stackrel{\vec{m}}{m}$ & $\begin{array}{l}\overrightarrow{\text { aे }} \\
\text { ה. }\end{array}$ & $\begin{array}{l}\infty \\
\stackrel{\infty}{i} \\
m\end{array}$ & $\stackrel{\circ}{\dot{a}}$ & $\begin{array}{c}m \\
\infty \\
\infty \\
\infty \\
N\end{array}$ & $\begin{array}{l}\stackrel{a}{\infty} \\
\stackrel{\infty}{+}\end{array}$ & ت্ & $\begin{array}{l}0 \\
\ddot{Q} \\
\ddot{i}\end{array}$ & $\begin{array}{l}\infty \\
\infty \\
\infty \\
\infty \\
\sim\end{array}$ & $\begin{array}{l}\infty \\
\text { i } \\
\text { ते }\end{array}$ & $\stackrel{\infty}{\dot{j}}$ & \begin{tabular}{l}
$\hat{0}$ \\
$\infty$ \\
\multirow{N}{N}{}
\end{tabular} & $\begin{array}{l}\hat{0} \\
\hat{N} \\
\stackrel{N}{N}\end{array}$ \\
\hline 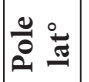 & $\stackrel{\substack{n \\
\infty}}{\stackrel{m}{m}}$ & $\begin{array}{l}\infty \\
\text { ஸे }\end{array}$ & $\begin{array}{l}\text {. } \\
\stackrel{\infty}{\sim}\end{array}$ & 苞 & $\stackrel{m}{\text { mे }}$ & $\begin{array}{l}\infty \\
\infty \\
\infty \\
\sim\end{array}$ & $\stackrel{\circ}{\circ}$ & $\vec{\infty}$ & $\begin{array}{l}0 \\
\infty \\
0 \\
0\end{array}$ & $\stackrel{\circ}{\stackrel{i}{N}}$ & $\ddot{\infty}$ & 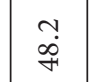 & $\underset{\infty}{+}$ & के & તై & $\begin{array}{l}\infty \\
\stackrel{q}{q}\end{array}$ \\
\hline 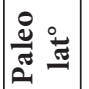 & $\overrightarrow{i n}$ & $\stackrel{\grave{q}}{\circ}$ & & $\hat{\vec{q}}$ & $\stackrel{\infty}{\stackrel{\rho}{\dot{m}}}$ & $\begin{array}{c}m \\
\substack{\infty \\
+}\end{array}$ & 亲 & $\begin{array}{c}m \\
\infty \\
\infty \\
+\end{array}$ & $\underset{m}{\infty}$ & 落 & $\stackrel{\circ}{\stackrel{4}{4}}$ & $\begin{array}{l}0 \\
\ddot{m}\end{array}$ & $\begin{array}{l}\infty \\
\stackrel{\leftrightarrow}{n}\end{array}$ & Oे & $\stackrel{\circ}{m}$ & $\stackrel{\sim}{\sim} \underset{\sim}{\sim}$ \\
\hline Fे & $\stackrel{\infty}{\underset{I}{\leftrightarrows}}$ & 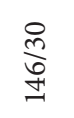 & $\stackrel{\hat{N}}{\stackrel{N}{a}}$ & 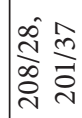 & 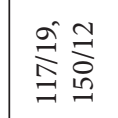 & 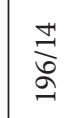 & 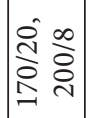 & 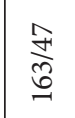 & $\underset{\infty}{\stackrel{0}{0}}$ & 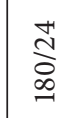 & $\underset{\vec{I}}{\vec{I}}$ & 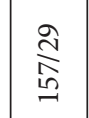 & $\begin{array}{l}\stackrel{n}{S} \\
\stackrel{\infty}{O} \\
\sim\end{array}$ & 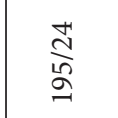 & 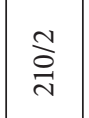 & $\underset{\substack{\infty \\
\infty}}{\stackrel{\infty}{=}}$ \\
\hline $\begin{array}{l}0^{n} \\
\gamma^{n}\end{array}$ & $\mathcal{I}$ & $a$ & $\stackrel{\sim}{\sim}$ & $n$ & $=$ & $n$ & in & 으 & $n$ & $\stackrel{\infty}{\sim}$ & $\stackrel{\sim}{N}$ & $\Xi$ & $\Rightarrow$ & 0 & 으 & $\underset{N}{ }$ \\
\hline 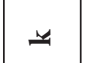 & $\stackrel{ }{0}$ & $\exists$ & 으 & $\stackrel{\sim}{\sim}$ & $\bar{m}$ & $\partial$ & $\stackrel{2}{2}$ & in & $\pi$ & 2 & ㅇ & i & $\stackrel{10}{\sim}$ & 今 & $F$ & $\exists$ \\
\hline 凷 & 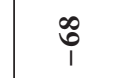 & $\begin{array}{l}\hat{0} \\
+\end{array}$ & $\begin{array}{l}8 \\
+ \\
+\end{array}$ & $\hat{i}$ & ip & $\begin{array}{l}\stackrel{0}{0} \\
+\end{array}$ & $\begin{array}{l}\vec{b} \\
+ \\
+\end{array}$ & $\begin{array}{l}\stackrel{8}{0} \\
+\end{array}$ & $\hat{i}$ & $\underset{+}{\mathbb{T}}$ & 草 & $\hat{p}$ & $\underset{+}{+}$ & i & $\hat{p}$ & F \\
\hline 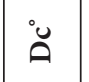 & J゙ & : & 衣 & $\Xi$ & $\cong$ & $\begin{array}{l}\infty \\
\stackrel{\leftrightarrow}{n}\end{array}$ & ల్ల & $\stackrel{f}{n}$ & $\stackrel{5}{\sigma}$ & 今 & $\vec{n}$ & $\widehat{\Xi}$ & $\stackrel{m}{m}$ & $\stackrel{\widetilde{\Xi}}{\sim}$ & I & $\stackrel{\text { I }}{=}$ \\
\hline $\begin{array}{l}0^{n} \\
\delta^{n}\end{array}$ & $\simeq$ & $a$ & $\stackrel{\sim}{\sim}$ & $n$ & $=$ & $\wedge$ & in & $\stackrel{\circ}{\circ}$ & $\wedge$ & $\stackrel{\infty}{\rightarrow}$ & $\stackrel{\sim}{\sim}$ & $\Xi$ & $=$ & 0 & $\circ$ & $\stackrel{\sim}{\sim}$ \\
\hline 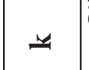 & $\stackrel{0}{\sim}$ & $\exists$ & 으 & $\stackrel{\sim}{\sim}$ & $\vec{m}$ & बे & $\kappa$ & in & $\nabla$ & 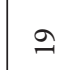 & 으 & i & $\stackrel{\mathscr{n}}{\sim}$ & f & $\exists$ & $\Rightarrow$ \\
\hline$\stackrel{-}{-}$ & $\tilde{\rho}$ & $\stackrel{\Re}{+}$ & 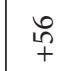 & $\vec{p}$ & $\stackrel{\sim}{p}$ & $\begin{array}{l}0 \\
+ \\
+\end{array}$ & $\underset{+}{+}$ & $\stackrel{\vartheta}{\mp}$ & $\widehat{\hat{1}}$ & $\begin{array}{l}\stackrel{0}{+} \\
+\end{array}$ & $\underset{⿱ 亠 巾 十}{+}$ & $\stackrel{\infty}{\uparrow}$ & $F$ & $\stackrel{m}{1}$ & (े & $r$ \\
\hline$\stackrel{\circ}{ }$ & 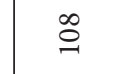 & $\underset{\sim}{\stackrel{D}{N}}$ & $\stackrel{m}{m}$ & 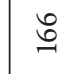 & $\stackrel{\infty}{=}$ & $\overline{\mathrm{N}}$ & ले & $\underset{d}{H}$ & 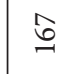 & $\vec{m}$ & $\widehat{m}$ & $\stackrel{\infty}{\sim}$ & in & 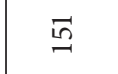 & $\stackrel{\infty}{\stackrel{\infty}{\sigma}}$ & తิ \\
\hline$\stackrel{?}{\sharp}$ & $\stackrel{\Xi}{\Xi}$ & $\stackrel{m}{\not}$ & $\frac{N}{i n}$ & $\stackrel{ }{\Xi}$ & 亲 & $\frac{H}{H}$ & $\stackrel{\beth}{\Xi}$ & $\hat{\sigma}$ & $\underset{\mathbb{N}}{\stackrel{2}{*}}$ & $\frac{0}{i n}$ & $\frac{\hat{b}}{0}$ & $\frac{N}{i n}$ & $\underset{\infty}{\stackrel{\sim}{*}}$ & $\underset{\stackrel{d}{d}}{\vec{d}}$ & $\frac{\infty}{n}$ & $\stackrel{9}{\frac{1}{1 n}}$ \\
\hline 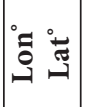 & 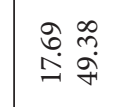 & 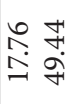 & 忩 & 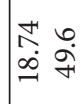 & 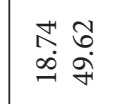 & 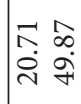 & 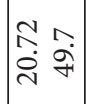 & 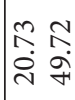 & $\begin{array}{l}\infty \\
\stackrel{N}{N} \\
\stackrel{i}{\sigma} \\
\dot{\sigma}\end{array}$ & 交 & 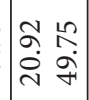 & 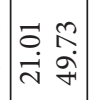 & 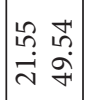 & $\begin{array}{l}\tilde{N} \\
\infty \\
\dot{\sim} \\
\dot{\sim}\end{array}$ & 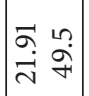 & 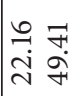 \\
\hline 落 & 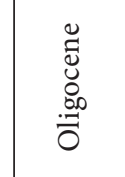 & 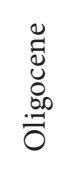 & $\begin{array}{l}: \\
\tilde{U} \\
\breve{.0} \\
.00 \\
07\end{array}$ & 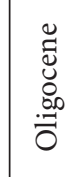 & 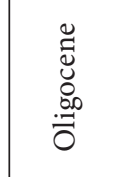 & 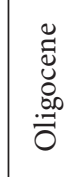 & 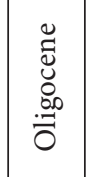 & 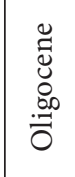 & 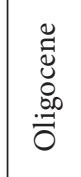 & 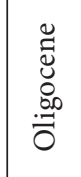 & 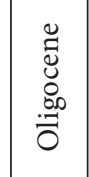 & 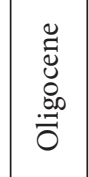 & 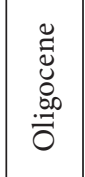 & 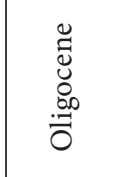 & 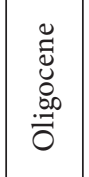 & 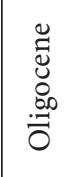 \\
\hline 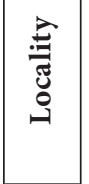 & 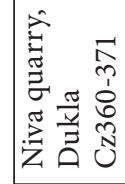 & 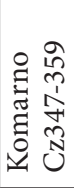 & 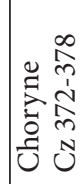 & 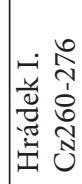 & 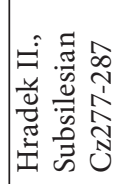 & 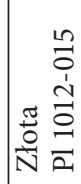 & 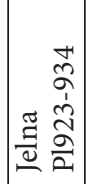 & 文 & 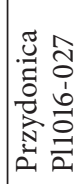 & 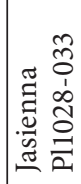 & 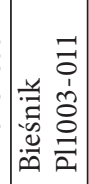 & 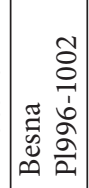 & 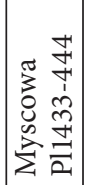 & 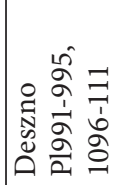 & 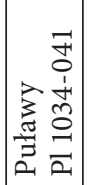 & 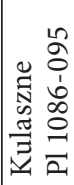 \\
\hline$\dot{z}$ & - & $\sim$ & $m$ & 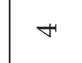 & in & 6 & $\wedge$ & $\infty$ & $a$ & 은 & $=$ & $\simeq$ & $\stackrel{m}{\longrightarrow}$ & $\exists$ & $\stackrel{n}{\sim}$ & $\stackrel{\circ}{\sim}$ \\
\hline
\end{tabular}




\begin{tabular}{|c|c|c|c|c|c|c|c|c|c|c|c|c|c|c|c|c|c|c|}
\hline$\ddot{\dddot{̋}}$ & $N$ & $\tau$ & - & - & - & - & $m$ & $m$ & $m$ & $m$ & $m$ & $r$ & $r$ & $r$ & 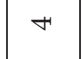 & $r$ & $r$ & $r$ \\
\hline$\stackrel{\circ}{\Xi}$ & $\stackrel{2}{N}$ & $\stackrel{\vartheta}{\varrho}$ & $\stackrel{亏}{\exists}$ & ָ̃ & $\vec{\infty}$ & $\curvearrowright$ & $\stackrel{+}{i}$ & $\stackrel{0}{+}$ & $\begin{array}{l}\infty \\
\stackrel{n}{ }\end{array}$ & $\underset{i}{0}$ & ڤْ & $\begin{array}{l}0 \\
\infty \\
\infty\end{array}$ & 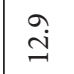 & in & $\stackrel{?}{+}$ & in & $\stackrel{\sim}{\exists}$ & ๙ั \\
\hline î & $\stackrel{\overbrace{}}{]}$ & $\stackrel{\wedge}{ \pm}$ & فํ. & $\begin{array}{l}\dot{d} \\
\dot{m}\end{array}$ & $\stackrel{8}{0}$ & $\exists$ & $\stackrel{\circ}{\circ}$ & فْ & $\ddot{\sim}$ & $\stackrel{\leftrightarrow}{\stackrel{f}{ }}$ & $\stackrel{9}{\exists}$ & $\stackrel{\circ}{\exists}$ & $\stackrel{9}{9}$ & $\stackrel{\infty}{\infty}_{\infty}^{0}$ & $\overbrace{\infty}^{m}$ & $\stackrel{N}{N}$ & $\stackrel{\sim}{ \pm}$ & $\stackrel{n}{0}$ \\
\hline \begin{tabular}{ll}
0 \\
\hdashline \\
\hdashline
\end{tabular} & $\stackrel{\infty}{i}$ & $\underset{\stackrel{D}{\sim}}{\stackrel{\vec{D}}{\sim}}$ & 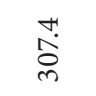 & $\begin{array}{l}\infty \\
\stackrel{\leftrightarrow}{\circ} \\
\text { ì }\end{array}$ & $\stackrel{\sim}{\tilde{m}}$ & $\begin{array}{l}\overrightarrow{0} \\
\dot{\infty} \\
\stackrel{0}{0}\end{array}$ & $\begin{array}{l}\infty \\
\stackrel{\infty}{\infty} \\
\sim \\
\sim\end{array}$ & $\overrightarrow{\tilde{d}}$ & $\begin{array}{l}\text { n̊. } \\
\text { Oें }\end{array}$ & $\begin{array}{l}\ddot{\leftrightarrow} \\
\stackrel{\leftrightarrow}{n}\end{array}$ & $\begin{array}{l}\overrightarrow{\mathrm{N}} \\
\overrightarrow{\mathrm{N}}\end{array}$ & $\begin{array}{l}\stackrel{0}{\circ} \\
\stackrel{m}{m}\end{array}$ & $\begin{array}{l}\stackrel{0}{0} \\
\stackrel{m}{m}\end{array}$ & 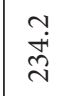 & $\begin{array}{l}\dot{0} \\
\dot{\Xi} \\
\dot{\sim}\end{array}$ & 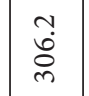 & $\begin{array}{l}\tilde{n} \\
\stackrel{n}{m}\end{array}$ & ָ̊. \\
\hline 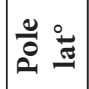 & $\stackrel{\circ}{\partial}$ & $\stackrel{\circ}{\dot{b}}$ & $\overrightarrow{6}$ & $\begin{array}{l}0 \\
\stackrel{\text { tin }}{i n}\end{array}$ & $\vec{b}$ & 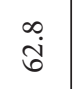 & $\begin{array}{c}n \\
\infty \\
\infty \\
+1\end{array}$ & $\hat{\vec{b}}$ & $\overrightarrow{\mathrm{J}}$ & 字 & ڤิં & $\hat{\mathscr{b}}$ & ڤें & 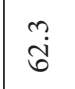 & in & ㅁ. & $\stackrel{\sim}{\mathscr{q}}$ & $\stackrel{?}{\stackrel{?}{*}}$ \\
\hline 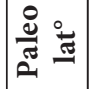 & $\stackrel{\text { İ }}{\mathrm{H}}$ & $\hat{\infty}$ & $\overrightarrow{\mathcal{H}}$ & $\begin{array}{l}\stackrel{0}{\circ} \\
\stackrel{m}{m}\end{array}$ & ֻุ. & 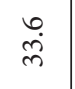 & $\stackrel{\wedge}{\stackrel{\Delta}{\sim}}$ & $\underset{\tilde{j}}{\tilde{j}}$ & $\stackrel{m}{\vec{\sim}}$ & $\stackrel{\stackrel{n}{g}}{\stackrel{\rightarrow}{g}}$ & $\stackrel{0}{\dot{\theta}}$ & $\stackrel{m}{+}$ & $\underset{\sim}{\stackrel{n}{i}}$ & 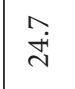 & $\ddot{\sigma}$ & ڤે̀ & $\stackrel{\infty}{\stackrel{\infty}{+}}$ & $\vec{\circ}$ \\
\hline छ & $\begin{array}{l}\infty \\
\stackrel{\infty}{\stackrel{N}{N}}\end{array}$ & 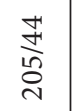 & 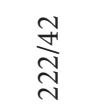 & $\frac{\stackrel{F}{\sigma}}{\stackrel{\Omega}{g}}$ & 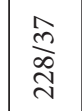 & $\frac{8}{\circ}$ & & & & & & 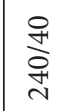 & 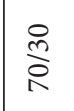 & $\stackrel{\infty}{\stackrel{\infty}{2}}$ & 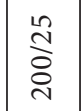 & 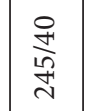 & 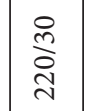 & 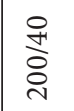 \\
\hline $\begin{array}{l}0 \\
\gamma^{n}\end{array}$ & 웅 & 으 & 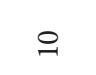 & $\vec{\sim}$ & in & $\infty$ & $\stackrel{\infty}{i}$ & in & $\stackrel{\infty}{2}$ & $\stackrel{+r}{+}$ & $\hat{\varrho}$ & n & $\Xi$ & $n$ & $\infty$ & in & $a$ & 0 \\
\hline$\simeq$ & 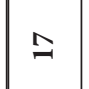 & $\stackrel{\sim}{\sim}$ & $\stackrel{n}{m}$ & $\exists$ & $\stackrel{\infty}{\circ}$ & F & ڤั. & $\begin{array}{l}\stackrel{H}{0} \\
\stackrel{+}{+}\end{array}$ & \begin{tabular}{l}
$\stackrel{\text { \} }{\mathrm{i}}} \\
{\text { (n) }}$ & $\underset{\vec{\dagger}}{\stackrel{\text { जे }}{4}}$ & $\stackrel{\infty}{\stackrel{\infty}{N}}$ & in & $=$ & $\mathscr{F}$ & నె & $\stackrel{\mathscr{0}}{\exists}$ & నె & 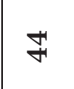 \\
\hline 凷 & $\underset{f}{+}$ & $\stackrel{\oplus}{+}$ & $\stackrel{\stackrel{m}{+}}{\stackrel{p}{n}}$ & $\stackrel{\circ}{+}$ & 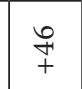 & $\stackrel{\infty}{+}$ & $\begin{array}{l}\stackrel{0}{0} \\
\stackrel{+}{1}\end{array}$ & $\stackrel{5}{\bar{p}}$ & 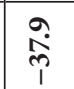 & iิ & ণิे & f & in & $\underset{f}{\mp}$ & $\overrightarrow{1}$ & $\stackrel{\infty}{\stackrel{\infty}{+}}$ & $\stackrel{\text { ț }}{+}$ & $\begin{array}{l}\bar{\sigma} \\
+\end{array}$ \\
\hline$\ddot{\breve{Q}}$ & $\frac{0}{m}$ & $\stackrel{\mathscr{D}}{\sim}$ & 芫 & $\underset{\sim}{\exists}$ & 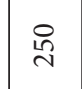 & $\stackrel{\infty}{m}$ & $\frac{9}{2}$ & $\stackrel{10}{3}$ & $\begin{array}{l}ت ّ \\
\stackrel{0}{~}\end{array}$ & 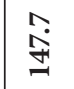 & ڤ̊. & $\stackrel{\overbrace{}}{\wedge}$ & $\stackrel{\varrho}{\exists}$ & $\stackrel{\infty}{\text { ஸे }}$ & $\stackrel{ }{\triangleq}$ & $\underset{⿱}{\mathbb{N}}$ & 芯 & $\underset{\sim}{\stackrel{P}{N}}$ \\
\hline $\begin{array}{l}0 \\
8^{\circ}\end{array}$ & 으 & 으 & 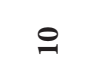 & $\bar{\sim}$ & in & $\infty$ & & & & & & $\wedge$ & $\exists$ & $\wedge$ & $\infty$ & in & $a$ & 6 \\
\hline 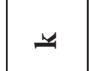 & 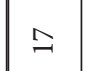 & $\stackrel{\text { i }}{ }$ & $\stackrel{m}{m}$ & $\Xi$ & $\stackrel{2}{\circ}$ & F & & & & & & in & $=$ & $\dddot{f}$ & ๗ె & 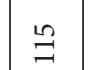 & లె & $\underset{\forall}{\not}$ \\
\hline i- & $\stackrel{\sim}{+}$ & $\stackrel{\infty}{\stackrel{\infty}{+}}$ & $\begin{array}{l}\overline{0} \\
+\end{array}$ & in & $\underset{+}{\stackrel{+}{+}}$ & $\stackrel{n}{+}$ & & & & & & $\begin{array}{l}\infty \\
0 \\
+\end{array}$ & $\hat{\imath}$ & $\underset{+}{\sharp}$ & $\infty$ & $\begin{array}{l}8 \\
0 \\
+\end{array}$ & $\begin{array}{l}20 \\
+\end{array}$ & $\begin{array}{l}n \\
+0 \\
+\end{array}$ \\
\hline$\stackrel{\circ}{\circ}$ & $\tilde{n}$ & స్ల & ڤั & $\frac{m}{m}$ & ల్లి & ల్ల & & & & & & m & ฉ & $\vec{m}$ & $\stackrel{\infty}{\stackrel{\infty}{\beth}}$ & $\stackrel{\infty}{m}$ & $\hat{m}$ & $\vec{m}$ \\
\hline 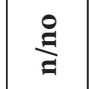 & $\underset{n}{m}$ & $\stackrel{n}{\varrho}$ & $\underset{\infty}{\stackrel{1}{\infty}}$ & $\frac{N}{i n}$ & $\underset{\Xi}{\Xi}$ & $\stackrel{乛}{a}$ & f & $\stackrel{m}{m}$ & $r$ & $\vec{n}$ & $\stackrel{\infty}{\sim}$ & $\underset{\Xi}{\Xi}$ & $\underset{\Xi}{\stackrel{J}{I}}$ & $\stackrel{\Xi}{\Xi}$ & $\underset{\mathrm{I}}{\stackrel{N}{I}}$ & $\underset{\infty}{\stackrel{\sim}{\infty}}$ & $\stackrel{\sim}{\stackrel{\Xi}{O}}$ & $\underset{⿱}{\stackrel{\Delta}{\sigma}}$ \\
\hline 品 & 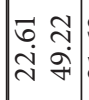 & 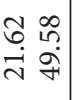 & 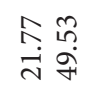 & 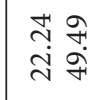 & 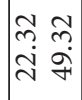 & 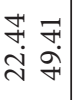 & 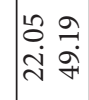 & 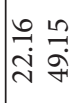 & 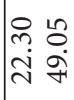 & 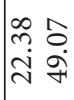 & $\begin{array}{l}\text { 우 } \\
\text { I } \\
\text { ì }\end{array}$ & 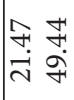 & 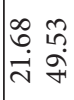 & 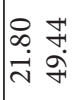 & $\begin{array}{l}\infty \\
\stackrel{F}{*} \\
\stackrel{\vec{\sigma}}{\vec{\sigma}}\end{array}$ & 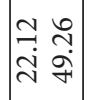 & 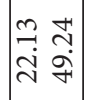 & 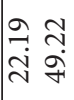 \\
\hline 总 & 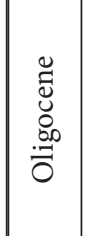 & 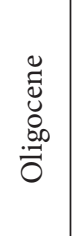 & 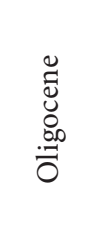 & 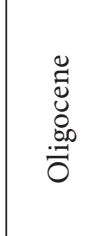 & 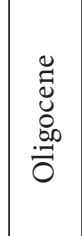 & 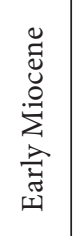 & 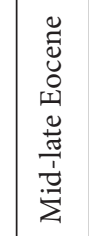 & 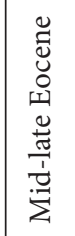 & 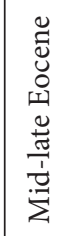 & 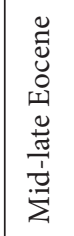 & 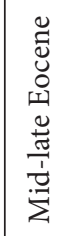 & 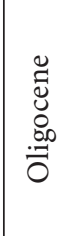 & 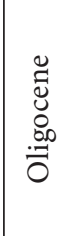 & 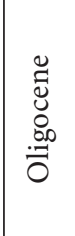 & 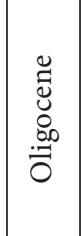 & 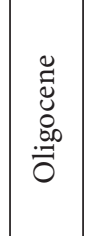 & 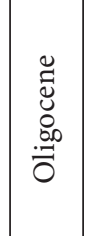 & 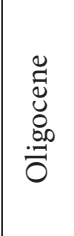 \\
\hline 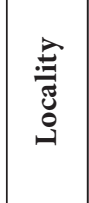 & 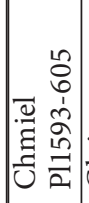 & 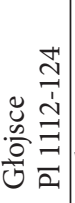 & 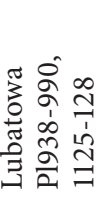 & 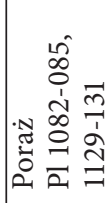 & 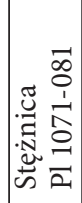 & 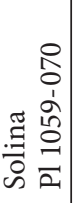 & 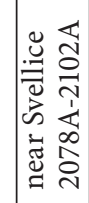 & 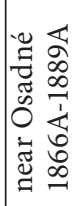 & 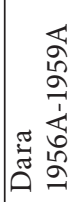 & 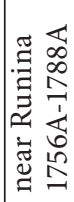 & 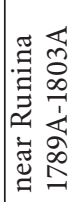 & 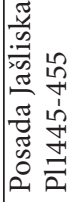 & 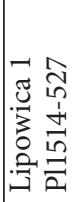 & 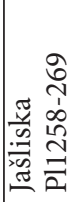 & 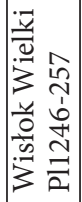 & 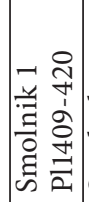 & 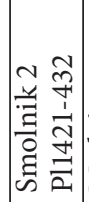 & 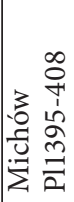 \\
\hline$\dot{\mathbf{z}}$ & 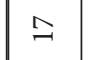 & $\stackrel{\infty}{\sim}$ & $\stackrel{2}{ }$ & ㄱ & $\vec{\sim}$ & $\tilde{N}$ & $\ddot{\sim}$ & $\stackrel{カ}{N}$ & $\stackrel{2}{\mathrm{~N}}$ & $\stackrel{\sim}{N}$ & $\hat{N}$ & $\stackrel{\infty}{\sim}$ & নे & $\stackrel{m}{m}$ & $\vec{m}$ & $\tilde{~}$ & $\stackrel{m}{m}$ & $\vec{m}$ \\
\hline
\end{tabular}
\end{tabular}




\begin{tabular}{|c|c|c|c|c|c|c|c|c|c|c|c|c|c|c|c|c|c|}
\hline & - & $\neg$ & $\neg$ & - & - & - & - & - & $\neg$ & - & - & - & - & in & in & in & in \\
\hline & $\stackrel{\circ}{a}$ & $\hat{\vec{m}}$ & $\exists$ & $\stackrel{\wedge}{\infty}$ & $\stackrel{ }{=}$ & $\widehat{\grave{m}}$ & $\stackrel{\wedge}{\infty}$ & $\stackrel{2}{N}$ & $\ddot{a}$ & $\stackrel{m}{\infty}$ & 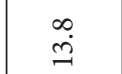 & $\stackrel{\rightleftarrows}{=}$ & $\stackrel{\circ}{9}$ & $\hat{m}$ & 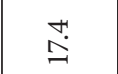 & $\begin{array}{l}\infty \\
\underset{j}{\mathrm{j}}\end{array}$ & $\underset{i}{\sim}$ \\
\hline & $\vec{\exists}$ & $\vec{\infty}$ & $\stackrel{9}{+}$ & $\hat{\mathrm{j}}$ & $\overrightarrow{\mathrm{i}}$ & ڤे & ते & $\vec{g}$ & $\stackrel{m}{\underset{I}{I}}$ & $\stackrel{\text { İ }}{\text { s. }}$ & $\stackrel{\rho}{\infty}$ & $\vec{n}$ & సे & in & $\stackrel{m}{\vec{N}}$ & $\stackrel{\wedge}{\infty}$ & $\stackrel{\circ}{+}$ \\
\hline & 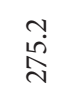 & $\begin{array}{l}\stackrel{0}{0} \\
\text { هे }\end{array}$ & 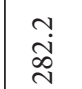 & $\overrightarrow{\vec{\omega}}$ & $\begin{array}{l}\text { mె. } \\
\text { bn } \\
\text { nd }\end{array}$ & $\begin{array}{l}0 \\
\text { ه̊. } \\
\text { in }\end{array}$ & $\begin{array}{l}\stackrel{\infty}{\grave{m}} \\
\stackrel{\dot{m}}{2}\end{array}$ & $\begin{array}{l}\stackrel{n}{2} \\
\stackrel{+}{\sim}\end{array}$ & ֶై & 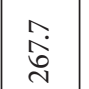 & $\begin{array}{l}\text { Pִ } \\
\vec{j}\end{array}$ & $\begin{array}{l}\infty \\
\stackrel{m}{m}\end{array}$ & $\begin{array}{l}\infty \\
\stackrel{\infty}{+}\end{array}$ & $\begin{array}{l}\stackrel{\dot{\alpha}}{\text { Oे }} \\
\text { }\end{array}$ & 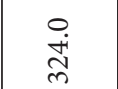 & ֶ̊: & 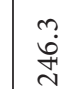 \\
\hline & $\begin{array}{l}\stackrel{0}{\infty} \\
\dot{\phi}\end{array}$ & $\vec{n}$ & $\widehat{\widehat{\sigma}}$ & $\stackrel{\hat{f}}{\dot{f}}$ & $\vec{b}$ & $\overrightarrow{\text { in }}$ & $\stackrel{\curvearrowright}{\not}$ & $\stackrel{\sim}{\stackrel{\sim}{*}}$ & $\begin{array}{l}\infty \\
\infty \\
\infty\end{array}$ & 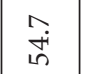 & $\vec{F}$ & बें & $\begin{array}{l}n \\
\infty \\
\infty\end{array}$ & $\stackrel{\curvearrowright}{\stackrel{\sim}{*}}$ & $\stackrel{\infty}{\stackrel{\infty}{+\infty}}$ & $\begin{array}{l}n \\
\infty \\
\infty \\
\infty\end{array}$ & ت્ \\
\hline & $\stackrel{+}{\stackrel{\sim}{\sim}}$ & ô & $\begin{array}{l}\infty \\
\stackrel{m}{\dot{m}}\end{array}$ & $\begin{array}{c}m \\
\infty \\
\infty \\
\infty\end{array}$ & $\stackrel{\hat{\sigma}}{ }$ & $\stackrel{\circ}{\stackrel{\sim}{0}}$ & $\begin{array}{l}m \\
\infty \\
\infty \\
+\end{array}$ & $\underset{\nexists}{ \pm}$ & $\overrightarrow{\widetilde{H}}$ & $\stackrel{\substack{\sim \\
\sim}}{\sim}$ & $\begin{array}{l}\stackrel{0}{0} \\
\text { m. }\end{array}$ & ồ & $\stackrel{\curvearrowright}{\dot{q}}$ & $\begin{array}{l}\text { } \\
\text { }\end{array}$ & $\begin{array}{l}m \\
\infty \\
\infty \\
+\end{array}$ & \&े & ळे \\
\hline & $\frac{n}{20}$ & 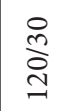 & $\frac{m}{\frac{n}{n}}$ & 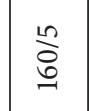 & 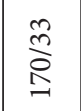 & 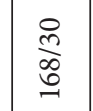 & 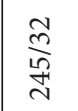 & $\underset{\substack{N \\
N}}{\stackrel{N}{n}}$ & $\begin{array}{l}\text { oे } \\
\text { d } \\
\text { v }\end{array}$ & 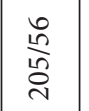 & 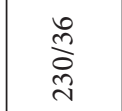 & 品 & 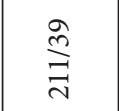 & 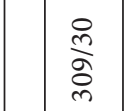 & 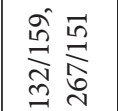 & $\stackrel{8}{\stackrel{0}{0}}$ & 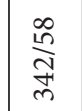 \\
\hline & $=$ & $\simeq$ & 으 & $\exists$ & $\stackrel{\infty}{\stackrel{\infty}{2}}$ & 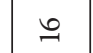 & $\Xi$ & $\simeq$ & $\infty$ & $\stackrel{9}{ }$ & $\stackrel{m}{=}$ & $\stackrel{\circ}{ }$ & $\stackrel{2}{\sim}$ & $r$ & $\stackrel{\sim}{\sim}$ & $\stackrel{\infty}{\infty}$ & $m$ \\
\hline & $\vec{n}$ & 우 & $\hat{n}$ & $\vec{m}$ & $\stackrel{\infty}{\sim}$ & $\exists$ & $F$ & 우 & 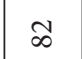 & $\vec{m}$ & $\hat{m}$ & $\stackrel{H}{F}$ & $\stackrel{\sim}{\sim}$ & $\underset{\sim}{\sim}$ & 6 & $\infty$ & $:$ \\
\hline & $\stackrel{\overbrace{}}{+}$ & $\underset{\infty}{\infty}$ & $\underset{⿱ 亠 巾}{+}$ & ô & $\stackrel{\infty}{\stackrel{\infty}{+}}$ & $\stackrel{?}{\stackrel{9}{+}}$ & $\stackrel{\widehat{m}}{+}$ & i & î & $\stackrel{7}{+}$ & $\widetilde{\sim}$ & $\stackrel{\overbrace{}}{+}$ & $\widehat{\overbrace{}}$ & î & ô & $\begin{array}{l}\text { t゙ } \\
+\end{array}$ & $\stackrel{q}{t}$ \\
\hline & $\frac{n}{m}$ & 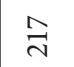 & శ్లి & 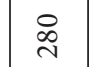 & ర్లి & $\stackrel{\text { H }}{\text { N }}$ & ֶై & $\sqrt{n}$ & กิ & : & 芯 & $\stackrel{\infty}{\underset{N}{N}}$ & $\underset{\sim}{\mathbb{N}}$ & $\bar{\Xi}$ & 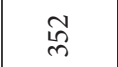 & $\frac{m}{m}$ & ले \\
\hline & $=$ & $\approx$ & 웅 & $\Xi$ & $\stackrel{\infty}{\sim}$ & 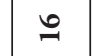 & $\Xi$ & $\approx$ & $\infty$ & 웅 & $\mathscr{2}$ & 욱 & 10 & $r$ & 9 & $\stackrel{\infty}{=}$ & $m$ \\
\hline & $\pi$ & q & nิ & $\bar{m}$ & $\stackrel{\infty}{\sim}$ & $\Xi$ & $F$ & 우 & ळూ & $\bar{n}$ & ले & $\exists$ & i & 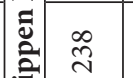 & 로 & $\infty$ & 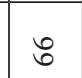 \\
\hline & $\stackrel{?}{\ddagger}$ & : & ถิ & $\begin{array}{l}0 \\
\vdots \\
+\end{array}$ & $\vec{q}$ & $\stackrel{\infty}{+}$ & $\stackrel{0}{0}$ & $\stackrel{\infty}{\uparrow}$ & $\vec{b}$ & f & î & $\underset{0}{8}$ & $\begin{array}{l}\text { ț } \\
+\end{array}$ & $\vec{\infty}$ & $\ddot{0}$ & $\stackrel{\sim}{\hat{p}}$ & $\stackrel{\Re}{\stackrel{n}{+}}$ \\
\hline & $\frac{m}{m}$ & $\stackrel{\infty}{\mathbb{Z}}$ & $\underset{ల}{H}$ & ลิ & స్ల & $\overrightarrow{\mathrm{m}}$ & $\stackrel{\infty}{\infty}$ & Iे & 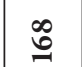 & $\stackrel{\sim}{\sim}$ & ลิ & $\underset{\sim}{\infty}$ & 古 & ஜ & $\sigma$ & ఫ্ల & $\underset{\bullet}{\infty}$ \\
\hline & $\stackrel{\mathscr{L}}{\Rightarrow}$ & in & $\stackrel{ }{\frac{1}{N}}$ & $\frac{1}{i n}$ & $\stackrel{\partial}{\partial}$ & 촛 & $\stackrel{\circ}{\underset{\gamma}{\gamma}}$ & $\frac{\partial}{n}$ & $\stackrel{\infty}{6}$ & 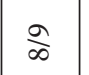 & $\frac{10}{i n}$ & $\frac{0}{6}$ & in & $\stackrel{m}{\frac{N}{N}}$ & $\frac{\alpha}{\infty}$ & $\stackrel{n}{\stackrel{2}{0}}$ & $\stackrel{0}{\stackrel{m}{m}}$ \\
\hline & 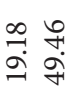 & $\begin{array}{l}\infty \\
\stackrel{F}{a} \\
\stackrel{F}{q}\end{array}$ & 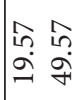 & 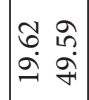 & 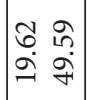 & $\left|\begin{array}{ll}2 & 0 \\
2 & \vdots \\
2 & \vdots \\
7\end{array}\right|$ & 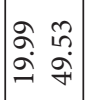 & 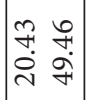 & 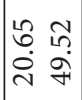 & 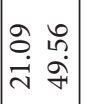 & 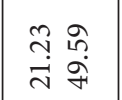 & 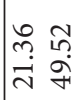 & 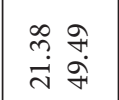 & 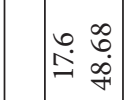 & $\begin{array}{l}\vec{f} \\
= \\
\infty\end{array}$ & $\left|\begin{array}{cc}0 & 0 \\
0 & 0 \\
0 & \dot{q}\end{array}\right|$ & $\vec{\partial}$ \\
\hline & 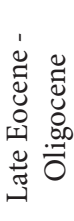 & 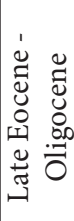 & 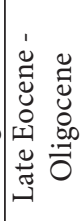 & 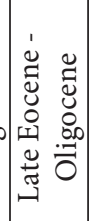 & 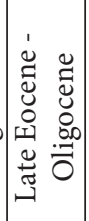 & 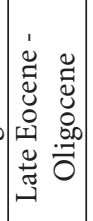 & 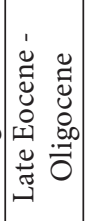 & 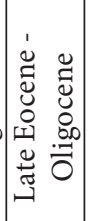 & 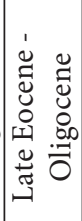 & 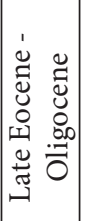 & 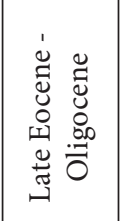 & 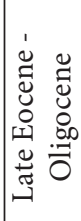 & 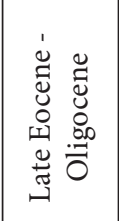 & 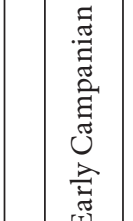 & 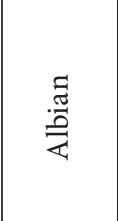 & 营 & 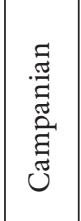 \\
\hline & 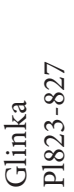 & 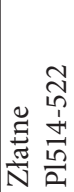 & 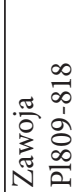 & 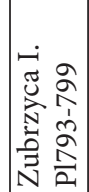 & 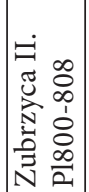 & 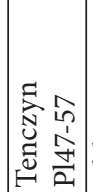 & 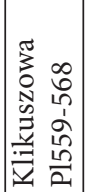 & 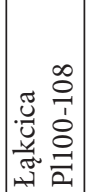 & 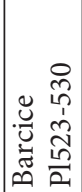 & 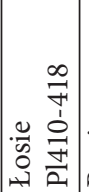 & 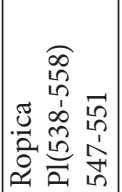 & 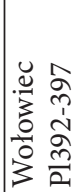 & 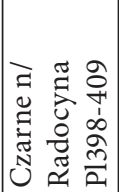 & 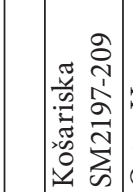 & 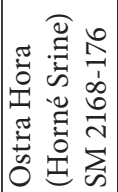 & 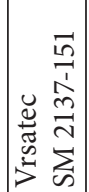 & 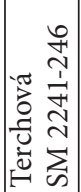 \\
\hline & $\stackrel{m}{m}$ & $\stackrel{ల}{\circ}$ & $\hat{m}$ & $\stackrel{\infty}{m}$ & ले & 아 & $F$ & 군 & $\dddot{F}$ & $\stackrel{H}{H}$ & $\mathscr{f}$ & \& & f & $\stackrel{\infty}{+}$ & Fे & in & in \\
\hline
\end{tabular}




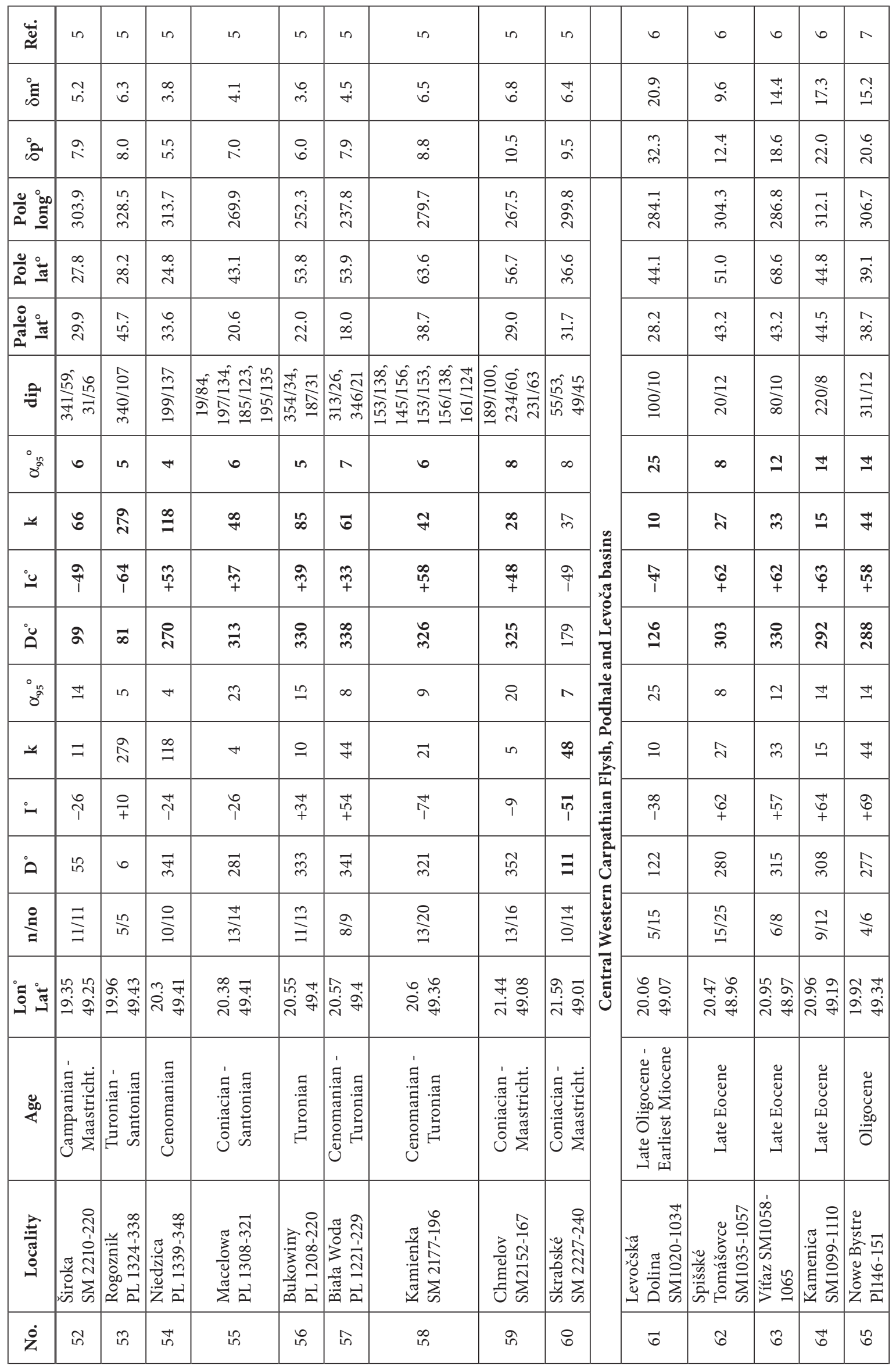




\begin{tabular}{|c|c|c|c|c|c|c|c|c|c|c|c|c|c|c|}
\hline$\wedge$ & $\wedge$ & $\wedge$ & $\wedge$ & $\wedge$ & $\infty$ & $\infty$ & $\infty$ & $\infty$ & $\infty$ & $\infty$ & $\infty$ & $\infty$ & $\infty$ & $\infty$ \\
\hline$\vec{m}$ & $\stackrel{?}{\underset{f}{f}}$ & $\vec{b}$ & 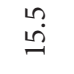 & $\vec{\exists}$ & Fे & $\stackrel{\sim}{m}$ & 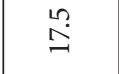 & $\stackrel{\leftrightarrow}{i}$ & $\hat{m}$ & $\begin{array}{l}\infty \\
\stackrel{\infty}{\infty}\end{array}$ & $\stackrel{\bullet}{\stackrel{\Xi}{\hookrightarrow}}$ & $\stackrel{\wedge}{\infty}$ & $\stackrel{\infty}{\stackrel{\infty}{+}}$ & فे \\
\hline$\stackrel{\not}{\infty}$ & $\underset{\vec{\sim}}{\stackrel{+}{*}}$ & ๙઼ & $\stackrel{\dot{\sim}}{\vec{\sim}}$ & $\stackrel{\partial}{\partial}$ & $\underset{\infty}{\infty}$ & $\overrightarrow{i n}$ & $\stackrel{\stackrel{+}{\sim}}{\sim}$ & $\vec{m}$ & $\ddot{H}$ & $\stackrel{\mathscr{L}}{\stackrel{+}{N}}$ & $\underset{\mathfrak{I}}{\stackrel{N}{N}}$ & $\stackrel{\sim}{\sim}$ & $\hat{b}$ & $\stackrel{0}{\dot{\varphi}}$ \\
\hline $\begin{array}{l}n \\
\hat{n} \\
\hat{n}\end{array}$ & $\begin{array}{l}\hat{b} \\
\stackrel{\infty}{\sim}\end{array}$ & $\vec{a}$ & $\begin{array}{l}0 \\
\text { +i } \\
\text { ì }\end{array}$ & $\begin{array}{l}a \\
\grave{\infty} \\
\stackrel{\sim}{0}\end{array}$ & $\stackrel{m}{\stackrel{0}{0}}$ & 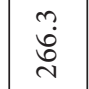 & 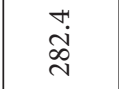 & 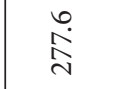 & $\underset{\substack{i \\
\sim}}{\stackrel{m}{d}}$ & $\underset{\sim}{\stackrel{+}{\sim}}$ & $\overrightarrow{\widetilde{N}}$ & $\begin{array}{l}\hat{i} \\
\hat{i}\end{array}$ & $\begin{array}{l}\ddot{0} \\
\dot{\varphi} \\
\ddot{n}\end{array}$ & $\overrightarrow{\stackrel{i}{i}}$ \\
\hline$\stackrel{\ddot{m}}{m}$ & 年 & $\begin{array}{l}\stackrel{\leftrightarrow}{\dot{f}} \\
\stackrel{4}{2}\end{array}$ & & $\widehat{\widehat{i}}$ & $\begin{array}{l}\infty \\
\stackrel{+}{+}\end{array}$ & $\hat{\mathrm{n}}$ & ลิ & $\stackrel{\circ}{\wedge}$ & m? & $\begin{array}{l}\stackrel{a}{d} \\
\stackrel{\infty}{ }\end{array}$ & ڤે & ஜ் & $\ddot{\text { तु }}$ & $\stackrel{H}{\text { in }}$ \\
\hline in & $\begin{array}{l}\infty \\
\stackrel{m}{m}\end{array}$ & बें & $\stackrel{\stackrel{m}{m}}{.}$ & $\begin{array}{l}i n \\
i n \\
m\end{array}$ & $\underset{\sim}{n}$ & $\begin{array}{l}\dot{0} \\
\dot{d}\end{array}$ & $\begin{array}{l}n ? \\
\text { bn }\end{array}$ & $\stackrel{\sim}{\stackrel{\leftrightarrow}{+}}$ & $\stackrel{\stackrel{m}{m}}{.}$ & $\overrightarrow{\mathcal{H}}$ & 萜 & নे & $\begin{array}{l}\dot{m} \\
\dot{m}\end{array}$ & $\begin{array}{l}0 \\
\dot{\infty}\end{array}$ \\
\hline$\underset{\mathrm{d}}{\stackrel{\mathrm{d}}{\mathrm{d}}}$ & 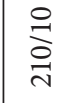 & 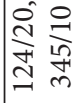 & 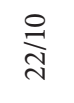 & $\begin{array}{l}\stackrel{\infty}{m} \\
\stackrel{0}{0}\end{array}$ & & & & & & & & & & \\
\hline 9 & $\stackrel{0}{\sim}$ & $n$ & $\beth$ & $\exists$ & $n$ & $r$ & $\beth$ & $N$ & $r$ & 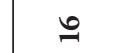 & $n$ & 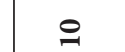 & in & $\infty$ \\
\hline$\stackrel{\sim}{N}$ & $\stackrel{0}{ }$ & $\stackrel{\infty}{\mathbb{N}}$ & নิ & 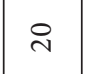 & ลิ & ڤิ & ిల & $\underset{\text { m }}{\text { m }}$ & $\stackrel{\bar{\sigma}}{g}$ & లి & $\hat{\sigma}$ & $\infty$ & oे & \\
\hline $\begin{array}{l}\stackrel{1}{6} \\
+\end{array}$ & $\stackrel{8}{\not 0}$ & $\stackrel{q}{+}$ & 후 & $\underset{+}{\stackrel{+}{+}}$ & $\stackrel{\infty}{\uparrow}$ & $\stackrel{q}{7}$ & ధ̊ & ర్ & $\stackrel{50}{+}$ & $\overrightarrow{0}$ & $\vec{i}$ & $\stackrel{q}{+}$ & $\hat{\imath}$ & $\begin{array}{c}\infty \\
\infty \\
+\end{array}$ \\
\hline 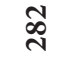 & 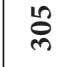 & స్ల & ลั & 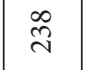 & I & $\mathcal{I}$ & ले & in & $\frac{\pi}{m}$ & $\infty$ & $\stackrel{\mathscr{I}}{\mathrm{I}}$ & గิ & ڤ્సે & 命 \\
\hline$\stackrel{m}{\rightarrow}$ & $\stackrel{0}{\circ}$ & $\stackrel{2}{2}$ & $\triangle$ & $\Xi$ & $\wedge$ & $r$ & $\bumpeq$ & $N$ & $r$ & $\stackrel{0}{0}$ & $n$ & 으 & in & $\infty$ \\
\hline$\stackrel{H}{\sim}$ & $\stackrel{0}{-1}$ & $\widehat{\imath}$ & $\grave{\lambda}$ & ิ & స̃ & బิ & ) & $\underset{\text { m }}{\text { m }}$ & $\stackrel{\overrightarrow{0}}{\exists}$ & $\stackrel{r}{n}$ & 6 & $\infty$ & :े & $\stackrel{\mathrm{N}}{ }$ \\
\hline$\stackrel{\infty}{\stackrel{\infty}{+}}$ & $\stackrel{\infty}{+}$ & $\stackrel{\text { Pr }}{+}$ & $\overrightarrow{b+}$ & $\stackrel{10}{+}$ & 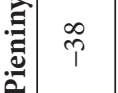 & $\stackrel{q}{7}$ & $\stackrel{0}{\ell}$ & $\begin{array}{l}\text { O } \\
\end{array}$ & $\overrightarrow{5 n}$ & $\vec{i}$ & $\vec{i}$ & $\stackrel{\vartheta}{+}$ & $\hat{p}$ & $\begin{array}{c}\infty \\
\infty \\
+ \\
+\end{array}$ \\
\hline$\underset{\sim}{\infty}$ & $\stackrel{b}{m}$ & ঐे & $\underset{\sim}{\stackrel{్}{\sim}}$ & $\frac{\pi}{m}$ & I & $\underset{I}{ }$ & ले & in & $\stackrel{\sim}{m}$ & $\infty$ & $\stackrel{\mathscr{L}}{\beth}$ & బ్ల & ڤे & 命 \\
\hline$\frac{\infty}{n}$ & $\stackrel{\frac{1}{6}}{6}$ & $\frac{10}{i n}$ & $\stackrel{\bigcirc}{\stackrel{\gamma}{\gamma}}$ & $\stackrel{\infty}{n}$ & $\frac{m}{m}$ & $\frac{m}{m}$ & $\frac{F}{r}$ & $\frac{\partial}{H}$ & $\frac{m}{m}$ & $\stackrel{n}{\not}$ & $\frac{\circ}{a}$ & $\stackrel{\vec{H}}{\not}$ & $\stackrel{F}{F}$ & $\frac{m}{m}$ \\
\hline $\begin{array}{ll}\tilde{c} & m \\
\dot{\sim} & \stackrel{\leftrightarrow}{\sigma}\end{array}$ & 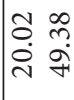 & 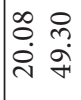 & 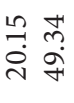 & 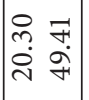 & 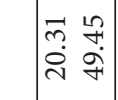 & 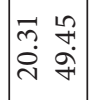 & 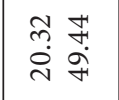 & 永 & 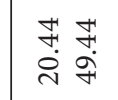 & 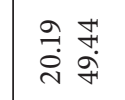 & 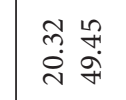 & 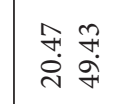 & 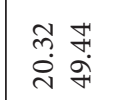 & 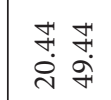 \\
\hline $\begin{array}{l}\mathscr{\Xi} \\
\breve{్} \\
\stackrel{0}{0} \\
: 0\end{array}$ & 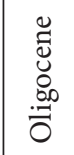 & 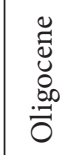 & 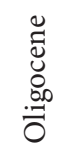 & 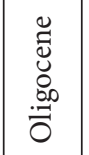 & 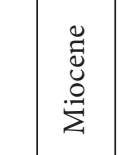 & 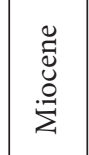 & 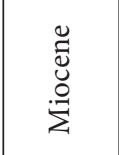 & 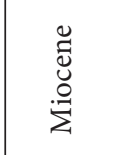 & 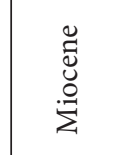 & 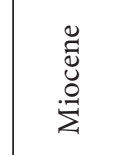 & $\begin{array}{l}\stackrel{\Xi}{\breve{~}} \\
\stackrel{\Xi}{\Sigma}\end{array}$ & 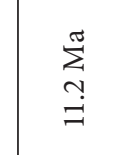 & $\begin{array}{l}\stackrel{\mathscr{U}}{~} \\
\stackrel{\mathscr{g}}{\Sigma}\end{array}$ & 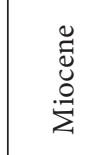 \\
\hline 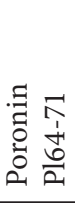 & 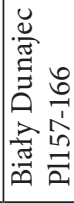 & 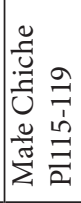 & 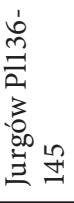 & 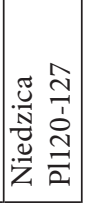 & 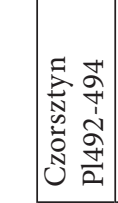 & 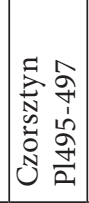 & 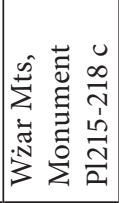 & 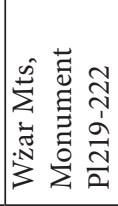 & 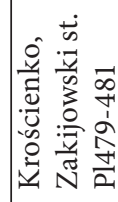 & 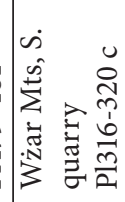 & 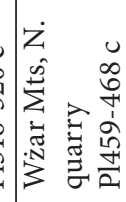 & 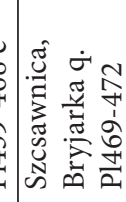 & 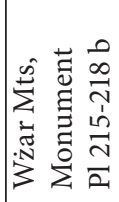 & 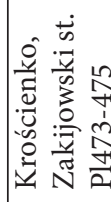 \\
\hline$\because$ & 6 & $\stackrel{\infty}{\circ}$ & a & $\stackrel{R}{R}$ & 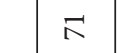 & 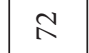 & 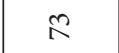 & ה & in & 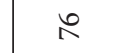 & 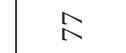 & $\stackrel{\infty}{\wedge}$ & $\curvearrowright$ & $\infty$ \\
\hline
\end{tabular}




\begin{tabular}{|c|c|c|c|c|c|c|c|c|c|c|c|c|c|c|c|c|c|}
\hline$\ddot{\widetilde{\Xi}}$ & $\infty$ & $\infty$ & $\infty$ & & $\begin{array}{l}\circ \\
a\end{array}$ & $\stackrel{ }{ }$ & $\begin{array}{l}\stackrel{2}{a} \\
a\end{array}$ & $\stackrel{\circ}{\circ}$ & 으 & $\stackrel{-}{\circ}$ & $\begin{array}{l}\circ \\
a\end{array}$ & $\stackrel{\circ}{\circ}$ & $=$ & $\exists$ & $\exists$ & $=$ & $=$ \\
\hline$\stackrel{\circ}{\Xi}$ & $\stackrel{\infty}{\infty}$ & $\hat{\vec{N}}$ & تే & & $\stackrel{\#}{\exists}$ & $\stackrel{\sim}{\varrho}$ & $\stackrel{\stackrel{n}{+}}{i}$ & $\hat{\varrho}$ & $\stackrel{\vartheta}{\varrho}$ & 官 & $\stackrel{\vec{J}}{\mathrm{~J}}$ & $\stackrel{\sim}{\sim}$ & 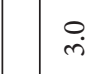 & $\stackrel{\circ}{=}$ & $\stackrel{\circ}{\mathrm{i}}$ & 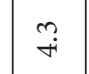 & $\stackrel{\sim}{+}$ \\
\hline î & $\stackrel{?}{0}$ & $\stackrel{\infty}{-i}$ & $\vec{\pi}$ & & $\stackrel{m}{ \pm}$ & $\stackrel{\hat{n}}{i}$ & $\underset{\infty}{\stackrel{\leftrightarrow}{\sim}}$ & $\stackrel{0}{\dot{\varphi}}$ & $\stackrel{\sim}{ \pm}$ & $\stackrel{m}{\sim}$ & $\stackrel{\sim}{n}$ & in. & $\stackrel{\infty}{\sim}$ & $\begin{array}{l}0 \\
\text { i }\end{array}$ & $\stackrel{+}{\mathrm{i}}$ & ๑. & ชู \\
\hline 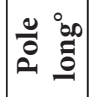 & กֶ. & $\stackrel{m}{a}$ & $\stackrel{0}{\ddot{\sim}}$ & & 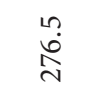 & 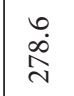 & $\stackrel{\circ}{\grave{m}}$ & $\stackrel{\circ}{\stackrel{i}{i}}$ & $\begin{array}{l}\text { n? } \\
\stackrel{0}{0}\end{array}$ & $\overrightarrow{\stackrel{0}{0}}$ & $\begin{array}{l}n \\
\infty \\
\infty \\
+\infty\end{array}$ & $\begin{array}{l}\circ \\
\dot{\circ}\end{array}$ & $\begin{array}{l}\infty \\
\stackrel{0}{9}\end{array}$ & $\begin{array}{l}\stackrel{\infty}{\stackrel{4}{m}} \\
\stackrel{m}{m}\end{array}$ & 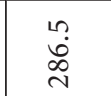 & $\stackrel{?}{\stackrel{n}{n}}$ & $\begin{array}{l}\stackrel{n}{n} \\
\stackrel{n}{N}\end{array}$ \\
\hline $\begin{array}{l}\frac{0}{0} \\
\stackrel{2}{*}\end{array}$ & $\begin{array}{l}\infty \\
\dot{\infty}\end{array}$ & 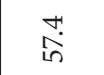 & $\stackrel{\circ}{\dot{\infty}}$ & & $\begin{array}{l}\infty \\
\infty \\
\infty \\
\infty\end{array}$ & $\begin{array}{l}n+0 \\
\infty \\
+\infty\end{array}$ & 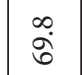 & ஜे & $\underset{N}{N}$ & $\vec{\circ}$ & $\stackrel{n}{0}$ & $\stackrel{\sim}{\infty}$ & $\begin{array}{l}\ddot{1} \\
\infty\end{array}$ & $\underset{\infty}{\infty}$ & $\underset{\infty}{\infty}$ & $\begin{array}{l}0 \\
i\end{array}$ & $\stackrel{N}{N}$ \\
\hline 递 & लें & 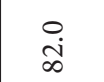 & $\begin{array}{l}0 \\
\infty \\
i \infty\end{array}$ & & is & ㅁ. & $\stackrel{n}{i}$ & $\stackrel{\infty}{\stackrel{m}{\dot{m}}}$ & 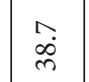 & $\begin{array}{l}\stackrel{0}{\mathrm{j}} \\
\text { m }\end{array}$ & 웅 & 官 & $\stackrel{m}{\not}$ & $\begin{array}{l}\infty \\
\text { in }\end{array}$ & ๙े & ָ̊. & $\stackrel{\vec{m}}{\vec{m}}$ \\
\hline$\vec{z}$ & & & & & 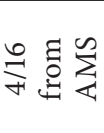 & 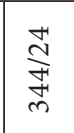 & 离 & $\stackrel{n}{2}$ & 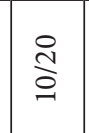 & $\stackrel{\circ}{\stackrel{i}{i}}$ & $\stackrel{\stackrel{\sim}{N}}{\stackrel{\infty}{d}}$ & 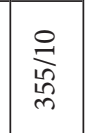 & 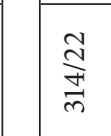 & $\stackrel{\stackrel{n}{1}}{\stackrel{\Delta}{a}}$ & 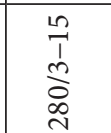 & $\frac{\widehat{a}}{\hat{\lambda}}$ & 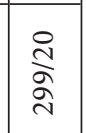 \\
\hline $\begin{array}{l}0 \\
8^{\circ}\end{array}$ & 6 & $\exists$ & $r$ & & $a$ & $\simeq$ & 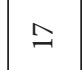 & $\simeq$ & 으 & $\triangle$ & $\wedge$ & $r$ & $\stackrel{+}{i}$ & $\stackrel{2}{\curvearrowright}$ & $\stackrel{10}{-}$ & $\stackrel{\sim}{\sim}$ & $\stackrel{0}{\mathscr{+}}$ \\
\hline$\underline{4}$ & $\stackrel{\mathscr{L}}{\not}$ & $\vec{n}$ & $\stackrel{0}{0}$ & & F & $\vec{\sim}$ & 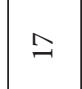 & $\mathscr{F}$ & $\widehat{へ}$ & নे & 8 & $\stackrel{b}{a}$ & $\begin{array}{l}0 \\
\dot{0} \\
0 \\
0\end{array}$ & $\hat{8}$ & تِ & $\stackrel{7}{\stackrel{0}{0}}$ & 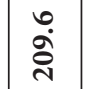 \\
\hline بِ & $\stackrel{?}{+}$ & $\begin{array}{l}\infty \\
\infty \\
1\end{array}$ & $\stackrel{\tilde{\imath}}{0}$ & & $\underset{+}{g}$ & $\stackrel{\widehat{T}}{+}$ & Fे & $\stackrel{\widehat{P}}{+}$ & $\stackrel{9}{+}$ & $\stackrel{0}{+1}$ & $\stackrel{n}{\stackrel{n}{+}}$ & $\stackrel{\text { f }}{+}$ & $\begin{array}{l}\hat{i} \\
+ \\
+\end{array}$ & $\underset{+}{\stackrel{4}{+}}$ & $\begin{array}{l}1 \\
10 \\
\vdots \\
+1\end{array}$ & 命 & 후 \\
\hline$\ddot{\circ}$ & $\stackrel{m}{2}$ & $\stackrel{\circlearrowright}{气}$ & $\stackrel{\infty}{\sim}$ & & $\vec{n}$ & శ్లి & ㅇ & ల్ల & 品 & 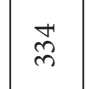 & ণิ & 0 & $\hat{o}$ & 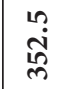 & 站 & 囟 & กั \\
\hline $0^{\circ}$ & 0 & $=$ & 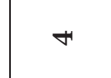 & & $a$ & $\simeq$ & $\beth$ & $\approx$ & 오 & $\triangle$ & $\wedge$ & 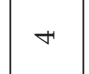 & $\stackrel{+}{i}$ & $\stackrel{?}{\wedge}$ & $\stackrel{9}{-}$ & $\stackrel{\sim}{\sim}$ & 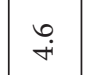 \\
\hline 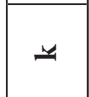 & 草 & in & $\stackrel{2}{2}$ & . & F & $\vec{\sim}$ & $\bumpeq$ & $\mathscr{F}$ & $\widehat{\curvearrowright}$ & নి & శి & $\stackrel{a}{\sigma}$ & 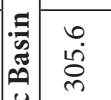 & $\hat{8}$ & $\begin{array}{l}0 \\
\infty \\
\stackrel{\infty}{9}\end{array}$ & 苛 & 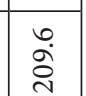 \\
\hline i- & $\stackrel{P}{+}$ & $\begin{array}{l}0 \\
\infty \\
1\end{array}$ & $\stackrel{n}{i}$ & Oี & $\begin{array}{l}\text { t゙ } \\
+\end{array}$ & $\stackrel{\infty}{+}$ & ô & $\stackrel{8}{10}$ & $\stackrel{\infty}{\stackrel{\infty}{+}}+$ & $\stackrel{\tilde{6}}{+}$ & $\stackrel{\infty}{\stackrel{\infty}{+}}$ & $\stackrel{\hat{p}}{+}$ & 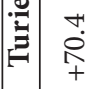 & $\underset{+}{\stackrel{Q}{+}}$ & $\begin{array}{l}0 \\
\stackrel{0}{0} \\
+ \\
+\end{array}$ & $\begin{array}{l}0 \\
\stackrel{0}{+} \\
+1\end{array}$ & $\begin{array}{l}H \\
\infty \\
\infty \\
+\end{array}$ \\
\hline$\stackrel{\circ}{\circ}$ & $\mathscr{O}$ & $\stackrel{0}{\cong}$ & $\stackrel{\infty}{\sim}$ & & 黑 & ले & $\stackrel{\infty}{\underset{J}{J}}$ & స్ల & 异 & స్లి & $\stackrel{\infty}{\sim}$ & - & $\underset{n}{\infty}$ & $\vec{\Lambda}$ & $\hat{\sigma}$ & $\stackrel{\curvearrowright}{\curvearrowright}$ & $\vec{\sim}$ \\
\hline$\stackrel{\stackrel{g}{\sharp}}{\sharp}$ & $\frac{m}{m}$ & $\frac{10}{10}$ & $\stackrel{9}{\circ}$ & & $\frac{m}{\infty}$ & $\frac{\infty}{\infty}$ & $\frac{1}{6}$ & $\frac{0}{i n}$ & à & $\stackrel{\bullet}{\not}$ & $\stackrel{2}{\infty}$ & $\frac{\partial}{\sigma}$ & $\underset{\sim}{\stackrel{n}{N}}$ & $\underset{\infty}{\vec{\sigma}}$ & $\underset{N}{\stackrel{N}{n}}$ & 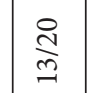 & $\frac{\infty}{6}$ \\
\hline 苛 & 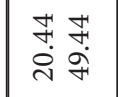 & 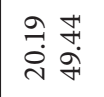 & 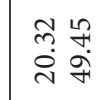 & & 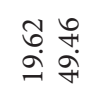 & $\begin{array}{ll}R & \hat{m} \\
\hat{m} & \stackrel{f}{\sigma}\end{array}$ & 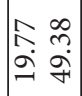 & 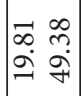 & 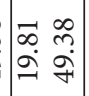 & 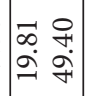 & 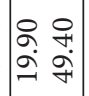 & $\mid$\begin{tabular}{ll}
0 & 0 \\
2 & + \\
\hdashline & $\vdots$ \\
\hdashline
\end{tabular} & 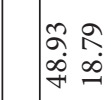 & 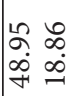 & 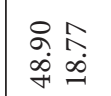 & $\left|\begin{array}{cc}0 & \infty \\
0 & \infty \\
\dot{q} & \infty \\
& 0\end{array}\right|$ & 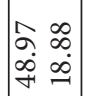 \\
\hline$\stackrel{8}{4}$ & 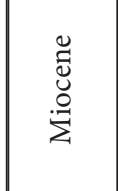 & $\begin{array}{l}\stackrel{\Xi}{\tilde{U}} \\
\stackrel{\Xi}{\Sigma}\end{array}$ & 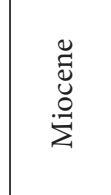 & & 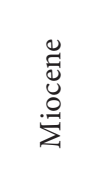 & $\begin{array}{l}\stackrel{\mathscr{\Xi}}{~} \\
\stackrel{\mathscr{g}}{\Sigma}\end{array}$ & $\begin{array}{l}\stackrel{\mathscr{\Xi}}{~} \\
\stackrel{\Xi}{\Sigma}\end{array}$ & 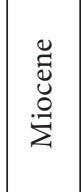 & 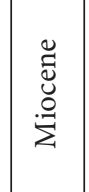 & 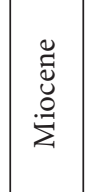 & $\begin{array}{l}\sum_{0}^{\pi} \\
= \\
=\end{array}$ & $\begin{array}{l}\sum_{0}^{\pi} \\
ٍ \\
=\end{array}$ & 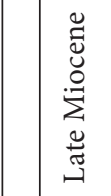 & 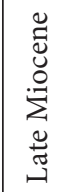 & 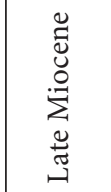 & 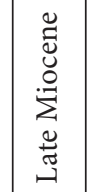 & 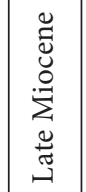 \\
\hline 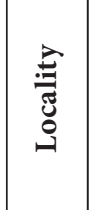 & 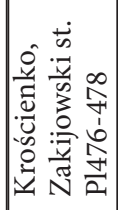 & 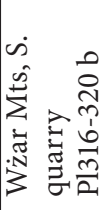 & 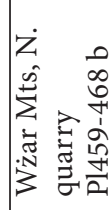 & & 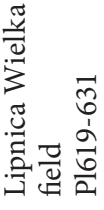 & 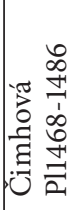 & 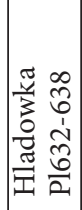 & 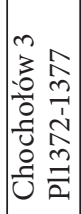 & 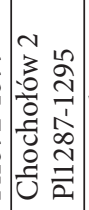 & 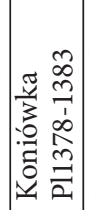 & 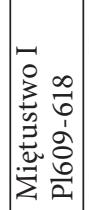 & 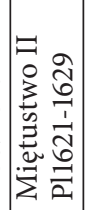 & 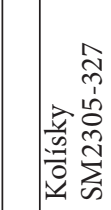 & 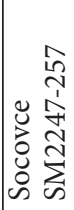 & 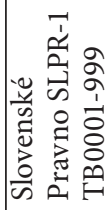 & 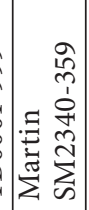 & 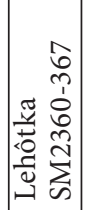 \\
\hline$\dot{\dot{z}}$ & $\vec{\infty}$ & $\infty_{\infty}$ & $\cong$ & & $\stackrel{\infty}{ }$ & $\stackrel{\mathscr{L}}{\infty}$ & 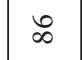 & $\infty$ & $\underset{\infty}{\infty}$ & ळे & \& & $\sigma$ & $\alpha$ & ๙ & J゙ & $\stackrel{2}{\alpha}$ & ஜ \\
\hline
\end{tabular}




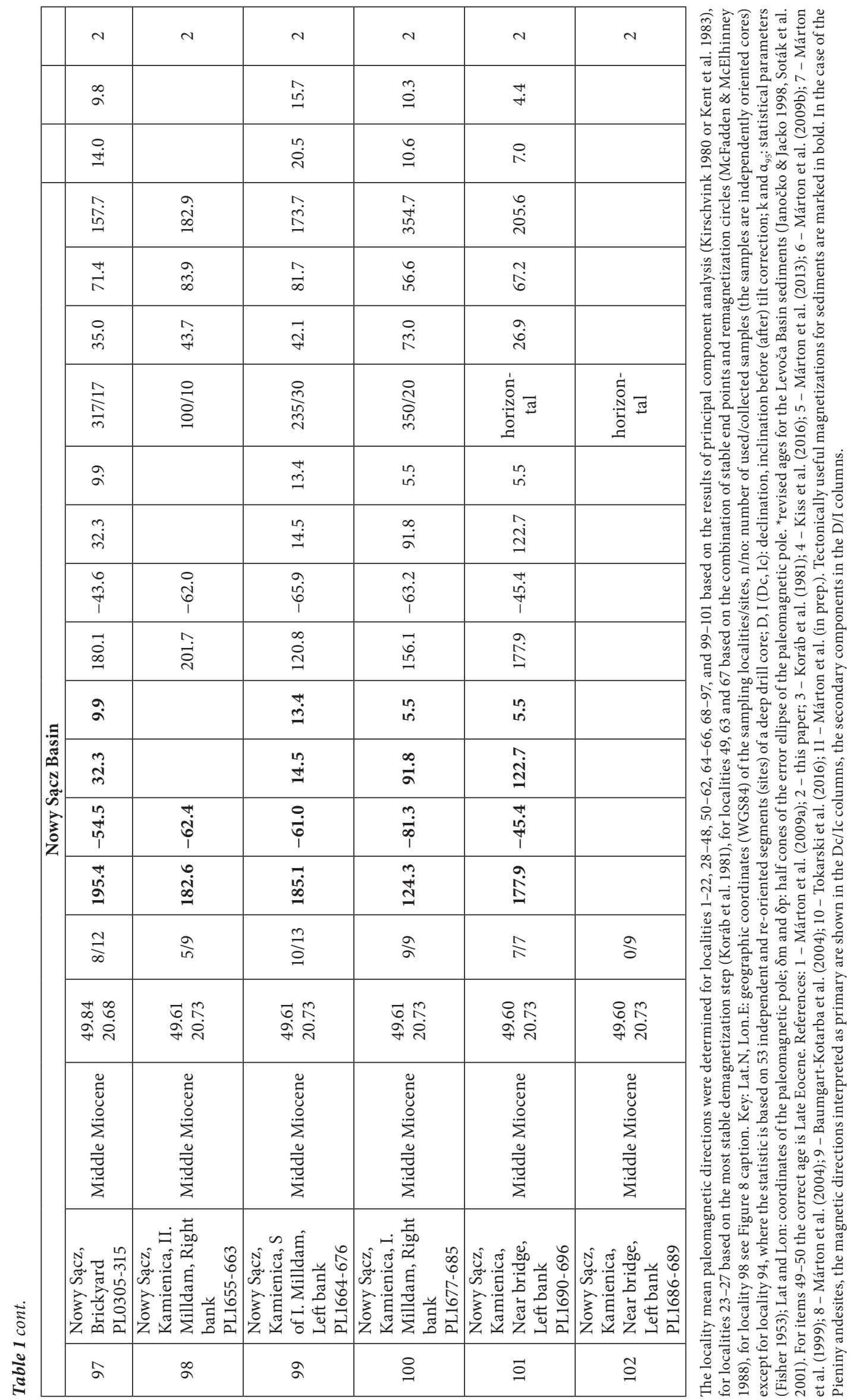




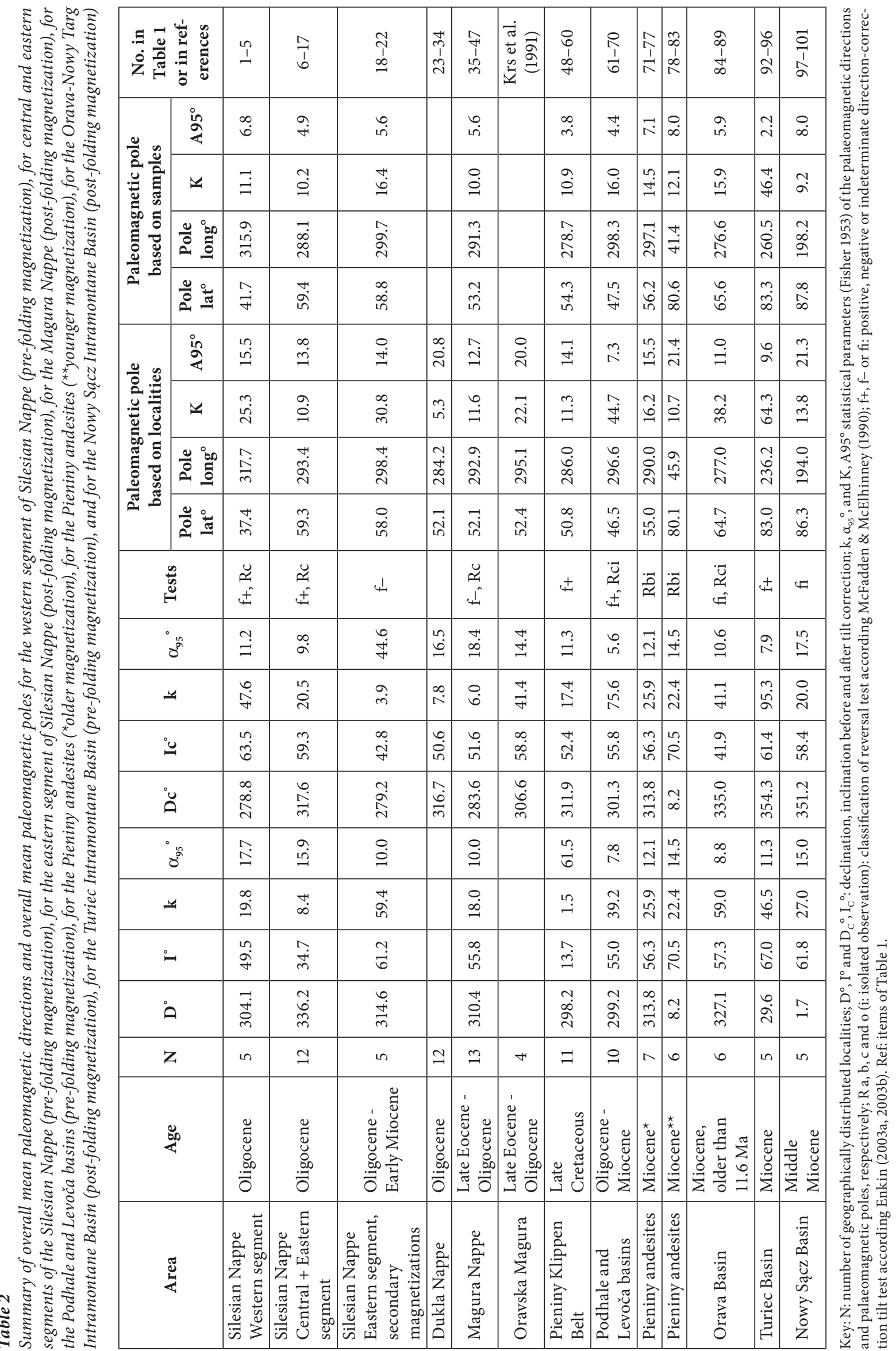




\section{GEOLOGICAL BACKGROUND}

During the Cenozoic, the Western Carpathians are subdivided into the Inner and the Outer Western Carpathians respectively, separated by the Pieniny Klippen Belt (Fig. 1), a highly tectonized narrow zone (e.g. Plašienka 2018 and references therein). The northern part of the Inner Western Carpathians is known as the Central Western Carpathians, which comprises three thick- and thinskinned nappe systems emplaced during the Late Cretaceous, namely the Tatric thick sheet overlain by the thin-skinned Fatric and Hronic units (e.g. Plašienka 2018). The nappe emplacement was followed by the deposition of an overstep sequence of flysch sediments in the Central Carpathian Paleogene Basin during the Late Eocene-Eearliest Miocene (e.g. Soták et al. 2001).

The Pieniny Klippen Belt belongs to a 3-5 km wide flower structure, limited by a fault zone cutting through the sediments of the Central Carpathian Paleogene basin in the south and the Magura Nappe in the north (Golonka et al. 2019). It is built up of Jurassic and Cretaceous sediments of extremely variable lithology. They were deposited in a paleogeographic realm comprising two basins separated by the Czorstyn ridge which was overridden by the accretionary prism of the Outer Western Carpathians during Late Cretaceous-Paleocene (Golonka et al. 2018). The destruction of this ridge led to the formation of submarine slumps and olistoliths in the Pieniny Klippen Belt and along the southern margin of the Magura Nappe (Golonka et al. 2015, Marzec et al. 2020), the innermost of the Outer Western Carpathian nappes (Fig. 1). The Jurassic and Lower Cretaceous sediments form the characteristic rigid blocks (klippen) which are embedded in the klippen mantle of Upper Cretaceous-Palogene marlstones, claystones and flysch (Birkenmajer 1986, Plašienka 2012). In the Miocene, the belt was intruded by andesites during two successive phases. The older set of intrusions in Poland is subparallel to the belt, while the younger one is NNW-SSE striking (Birkenmajer 1986). Some of the intrusions penetrate the Paleogene sediments of the Magura Nappe.

The fold and thrust belt of the Outer Western Carpathians is emplaced over the Miocene sediments of the Carpathian Foredeep which were deposited on the southern margin of stable Europe
(North European plate). The trust belt comprises the Magura, Dukla, Silesian, Subsilesian and Skole rootless nappes (Fig. 1), which are mainly composed of Upper Cretaceous-Paleogene flysch (e.g. Rakús 1998, Oszczypko 2006, Oszczypko \& Oszczypko-Clowes 2009a, Kováč et al. 2016). The nappe pile started to form during the Eocene (e.g. Świerczewska \& Tokarski 1998, Nemčok et al. 2006) and the deformation progressed from the hinterland towards north (in present coordinates). Two main phases of nappe stacking were recognized. The tectonic transport was directed towards NW (in present co-ordinates) during the first phase and towards NE during the second phase while the folds from the first phase were partly re-folded (Aleksandrowski 1985).

In the Miocene, several basins opened up in both the Outer and Inner Carpathians (for a comprehensive review see Kováč et al. 2017, 2018) such as the Rzeszów (not discussed in this paper to obtain paleomagnetic results from this basin), the Nowy Sącz (e.g. Oszczypko 1973, Oszczypko-Clowes et al. 2009b), the Orava-Nowy Targ (e.g. Baumgart-Kotarba et al. 2004, Tokarski et al. 2012, 2016, Loziński et al. 2015, Ludwiniak et al. 2019) and the Turiec intramontane basins (e.g. Kovač et al. 2011, Pipík et al. 2012). The intramontane depressions were filled by Neogene and Quaternary sediments of different character (conglomerates, sandstones, siltstones, marlstones) that deposited on land, in fresh or brackish water.

\section{MATERIALS AND METHODS}

As mentioned earlier, the majority of the paleomagnetic results reviewed here were published and the original papers documented the methods of field collecting and laboratory processing of the oriented samples as well as the statistical methods of evaluation, which permitted us to define the locality mean paleomagnetic directions (Tab. 1). The exception is the more recently studied the Nowy Sacz Basin, for which the documentation necessary to support the reliability of the data will be presented as follows.

Miocene sediments were collected from the Nowy Sącz Basin during two short field campaigns. In 2000, the samples were drilled from an active brickyard. In 2016, when both of the formerly existing brickyards were found abandoned and the 
sediments weathered, fresh samples were drilled from natural outcrops along the Kamenica River. The samples were oriented in situ with a magnetic compass, wrapped in aluminium and vacuum foil and kept in refrigerator in order to protect them from chemical alteration and disintegration.

In the laboratory, just before the paleomagnetic processing, standard-size specimens were cut from each sample. It was followed by the measurements of the natural remanent magnetization (NRM) in the natural state and that of the anisotropy of the magnetic susceptibility (AMS). The NRM was measured with JR-4 and JR-5A magnetometers, the AMS with a KLY-2 kappabridge. The specimens were subjected to stepwise thermal demagnetization until the NRM was completely demagnetized or the signal was lost due to instability. After each step, the NRM and the magnetic susceptibility was re-measured. The NRM was completely or near-completely demagnetized by $400^{\circ} \mathrm{C}$, while the magnetic susceptibility in most cases indicated the formation of magnetite suggesting that the original magnetic mineral in the studied sediments was greigite (Fig. 2). The demagnetization curves were analyzed for linear segments and the component decaying towards the origin of the Zijderveld diagrams (Fig. 2) was considered as the characteristic remanent magnetization. These components were used to compute the mean paleomagnetic directions for localities 97, and 99-101 (Tab. 1). For locality 98, statistical parameters were not tabulated. At this locality, the directions of the individual samples form a great circle and the locality mean paleomagnetic direction was obtained with the method proposed by McFadden \& McElhinney (1988). Nevertheless it is extremely useful in cases where stable magnetization characterizes some samples/localities while others exhibit great circle movement of the vector between the stable component of the first group and another component acquired at a different time and/or in different position of the strata.
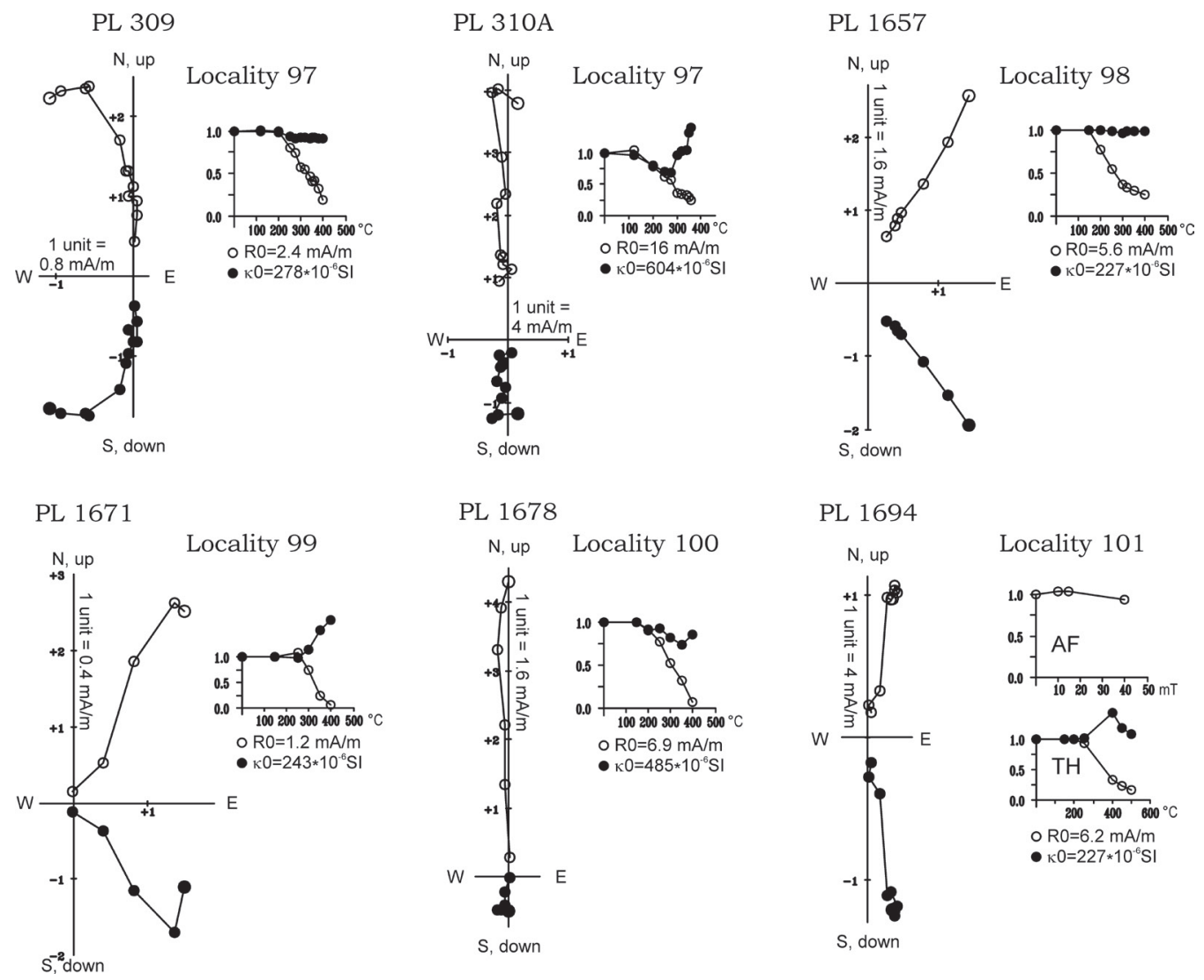

Locality 101
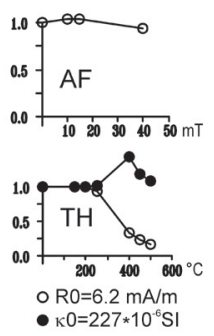

Fig. 2. Nowy Sacz Intramontane Basin. Upper Miocene-Pliocene sediments. Typical thermal demagnetization curves with susceptibility monitored during heating. For each specimen, the left side diagram shows the change of the direction of the NRM on heating (Zijderveld diagrams), the right side diagram the change of NRM intensity (hollow circles) and that of the low field magnetic susceptibility (dots) as a function of the temperature. In the case of specimen Pl1694, the demagnetization started with the AF method and continued with the thermal 
The age of the magnetization with respect to the stratigraphic age were constrained with a tilt test, using the local azimuth and dip of the sampled strata in the procedure. As the sediments were poorly indurated, the values measured in the field were compared to the orientation of the well-defined AMS foliation planes, which justified the tilt corrections for localities 97, 100 and 101, being sub-parallel to the bedding planes measured in the field. For the other localities, a comparison was not possible as the AMS fabric was chaotic.

The database of Table 1 contains 102 entries. For all of them, the relationship between the age of the source rock and its magnetization is indicated (pre or post-folding/tilting). The paleomagnetic directions to be considered in the tectonic interpretation are in bold, which in the case of sediments are either before (magnetization acquired after tilting) or after tilt correction (magnetization acquired before tilting). For most of the Pieniny andesites, two components of the NRM were identified. They are considered as reflecting the orientation of the area at different times.

Based on the entries of Table 1, overall mean paleomagnetic results were computed (Tab. 2) from quasi-coeval and geographically distributed localities representing a certain unit. These overall-mean paleomagnetic directions are used as constraints for large-scale displacements.

\section{DISCUSSION OF THE AVAILABLE PALEOMAGNETIC DATA}

As it is widely known, a paleo-inclination is a function of the latitude at which the magnetization was acquired (Fig. 3A). In contrast, a paleo-declination is the resultant of the rotations which took place after the acquisition of the magnetization (Fig. 3B, C).

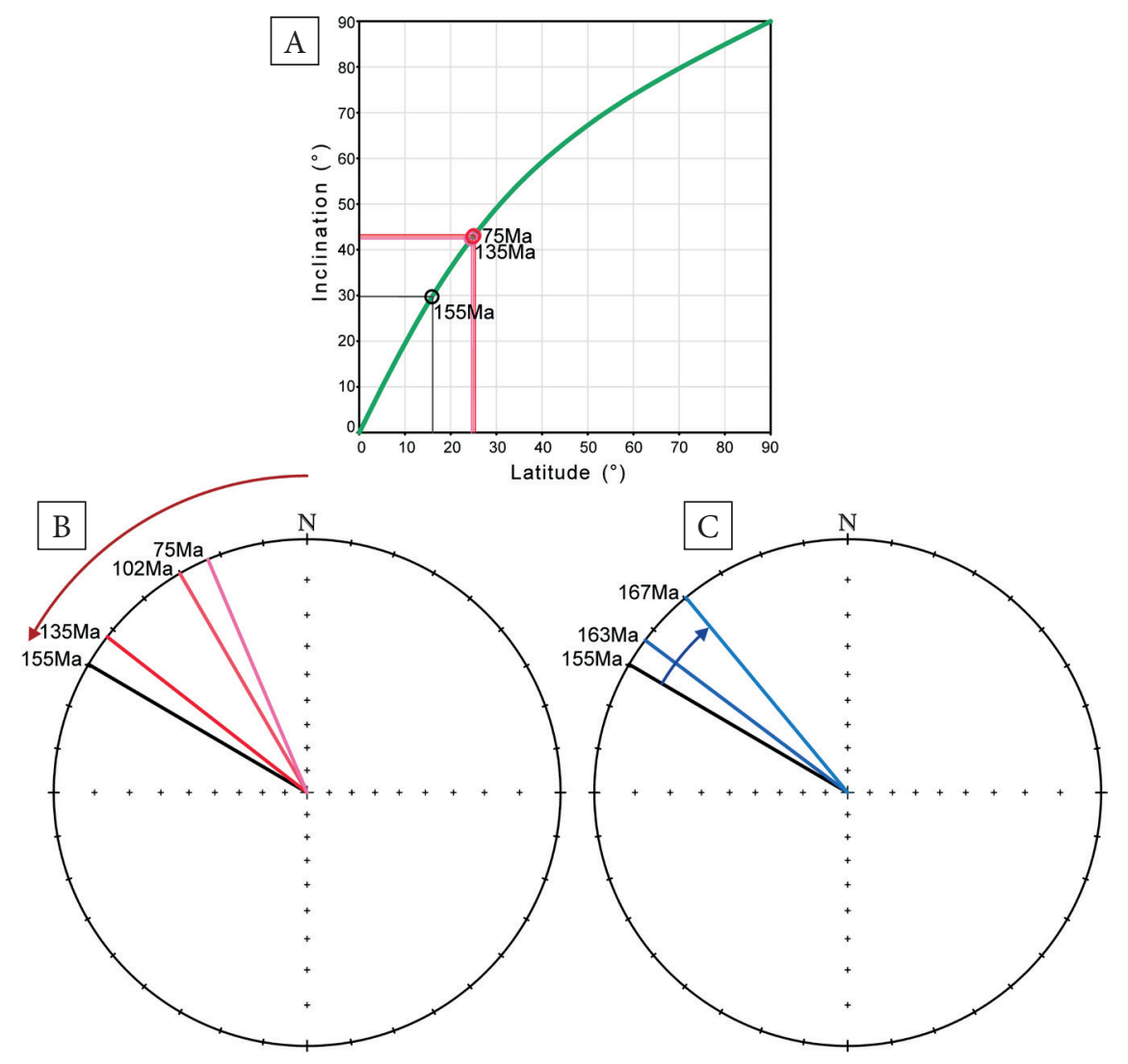

Fig. 3. Interpretation of paleomagnetic inclinations which constrain the latitude where the rocks acquired magnetizations (Fig. $3 A$ ) and the pattern of paleomagnetic declinations obtained on rocks of different ages suggesting CCW (Fig. $3 B$ ) and CW (Fig. 3C) rotations, respectively. The examples represent paleomagnetic results obtained from the northern part of stable Adria (Adriatic microplate, Márton et al. 2017). Figure $3 \mathrm{~A}$ shows that Adria must have been at about $16^{\circ} \mathrm{N}$ at $155 \mathrm{Ma}$ and about $25^{\circ} \mathrm{N}$ at 135-75 Ma. Figure 3B represents the steps of CCW rotation, which led to the maximum angle of the measured CCW rotation on rocks about $155 \mathrm{Ma}$ old. Figure 3C shows how the more moderate CCW rotations measured on rocks older than $155 \mathrm{Ma}$, which compared to the maximum angle of CCW rotation suggests CW rotations predating $155 \mathrm{Ma}$ 
It follows that the correct interpretation of a paleo-declination is easier for younger than older rocks from the same tectonic unit. That is why paleomagnetic data are particularly reliable constraints for the subject of the present paper.

A paleomagnetic result is generally considered valuable when the age of the source rock and its magnetization is the same, i.e. the latter can be related to the stratigraphic or isotope age of a rock unit. However, consistent secondary magnetizations e.g. during chemical alteration and/or elevated temperature, can also provide important paleomagnetic constraints, as it will be discussed below.

\section{Large scale rotations during the emplacement of the Outer Western Carpathian nappes over the European margin}

The flysch of Cenozoic age was studied at geographically distributed localities from the Outer Carpathian nappes (Fig. 1). No positive results were acquired from the Skola Nappe, although several points were tested. Concerning the other nappes, good results were obtained from the Western, Central and Eastern segments, respectively of the Silesian Nappe (Márton et al. 2009a), the Dukla Nappe (Koráb et al. 1981, Kiss et al. 2016), and the Magura Nappe (Márton et al. 2009a).

The paleomagnetic results suggest large-scale Miocene CCW rotation of the Silesian Nappe (Tab. 1). This vertical axis rotation must have post-dated the folding of the Paleogene (Lower Miocene) strata, since it is exhibited by all localities with pre- as well as localities with post-folding remanence (Tab. 2). The angle of the rotation is about $50^{\circ}$ (with respect to north) in the Central and Eastern segments and somewhat larger in the Western segment, probably due to post-nappe emplacement rotations within a left lateral wrench corridor (Fodor at al. 1995, Márton et al. 2009a). The $50^{\circ}$ rotation relative to north is about $60^{\circ}$ with respect to stable Europe, as the reference declination computed from the APW (Torsvik et al. 2012) for the time interval between 30 and $20 \mathrm{Ma}$ is $9.4-10.8^{\circ}$.

At this point it is worth mentioning that the Miocene sediments of the Carpathian Foredeep exhibit the expected stable European declination only as secondary with respect to their deformation. Meanwhile the magnetic signal predating the deformation, and measured (with a single exception) older than around $10 \mathrm{Ma}$ strata suggest about $20^{\circ} \mathrm{CCW}$ rotation with respect to the stable European reference declination (Scholger \& Stingl 2004, Márton et al. 2011). This result implies that the southern margin of stable Europe must have been affected by forces generated by the overthrusting nappe piles but does not invalidate the reference declinations computed from the European APW of Torsvik et al. (2012).

The paleomagnetic results from the Dukla Nappe represent both the Slovak (Koráb et al. 1981) and the Polish (Kiss et al. 2016) parts. Only tilt corrected paleomagnetic directions are available from the which lend themselves to a combination with tilt corrected ones from the second (Fig. 4). The individual paleomagnetic directions exhibit a smeared distribution in declination. This can be attributed, at least partly, to the melange character of the Oligocene sediments in the Polish sector of the Dukla Nappe (Siemińska et al. 2020). The distribution of the vectors prohibits the definition of an overall-mean paleomagnetic direction for the Dukla Nappe, yet the picture obviously suggests a general CCW rotation.

Two sets of paleomagnetic results are available from the Paleogene flysch of the Magura Nappe.

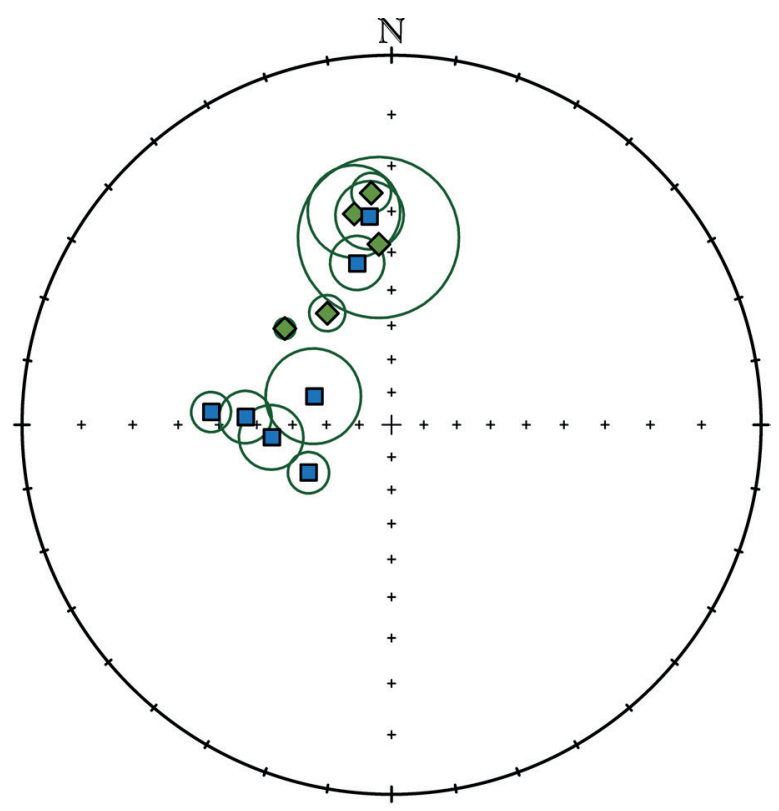

Fig. 4. Dukla Nappe. Tilt corrected paleomagnetic directions with $\alpha_{95}$ on an equal angle projection. Diamonds: Koráb et al. (1981), Squares: Kiss et al. (2016) 
The statistically loosely defined locality mean paleomagnetic directions by Krs et al. (1991) suggest large CCW rotation. Those by Márton et al. (2009a) are of sufficiently high statistical precision, thus permit the conclusion that the rotation took place after the folding of the strata (Tab. 2).

In the hinterland of the Outer Western Carpathians (Fig. 1), large post-Cretaceous CCW rotation was documented for the Pieniny Klippen Belt (Márton et al. 2013). The Late Eocene-Earliest Miocene flysch sediments (Filo \& Siráňová 1996, Gedl 1998, Janočko \& Jacko 1998, Janočko et al. 1998, Soták et al. 2001, Janočko 2002) of the Central Carpathian Paleogene Basin also show about $50^{\circ} \mathrm{CCW}$ vertical axis rotation with respect to the north (Márton et al. 1999, 2009b). The magnetizations in both units were acquired prior to folding/tilting (Tab. 2).

The similar magnitude and timing of the CCW rotation in the Outer and Central Western Carpathians (Márton et al. 2016) suggest that these units rotated in co-ordination and the movements naturally involved the Pieniny Klippen Belt (Fig. 5). This justifies the search for evidence for the termination of the large-scale CCW rotation in all three units.

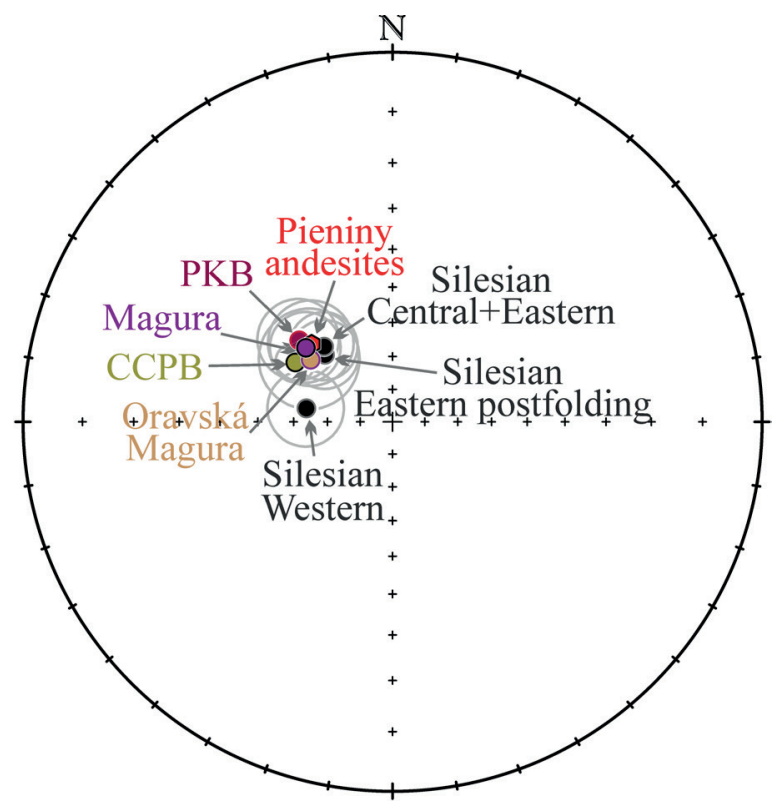

Fig. 5. Overall mean paleomagnetic directions with $\alpha_{95}$ (data from Table 2) are shown on equal angle projection. Note the extremely high consistency of the paleomagnetic declinations/inclinations, except the western segment of the Silesian Nappe, where the extra CCW rotation might be due to the left lateral shear affecting the area (Márton et al. 2009a). Key: PKB, Pieniny Klippen Belt; CCPB: Central Carpathian Paleogene Basin

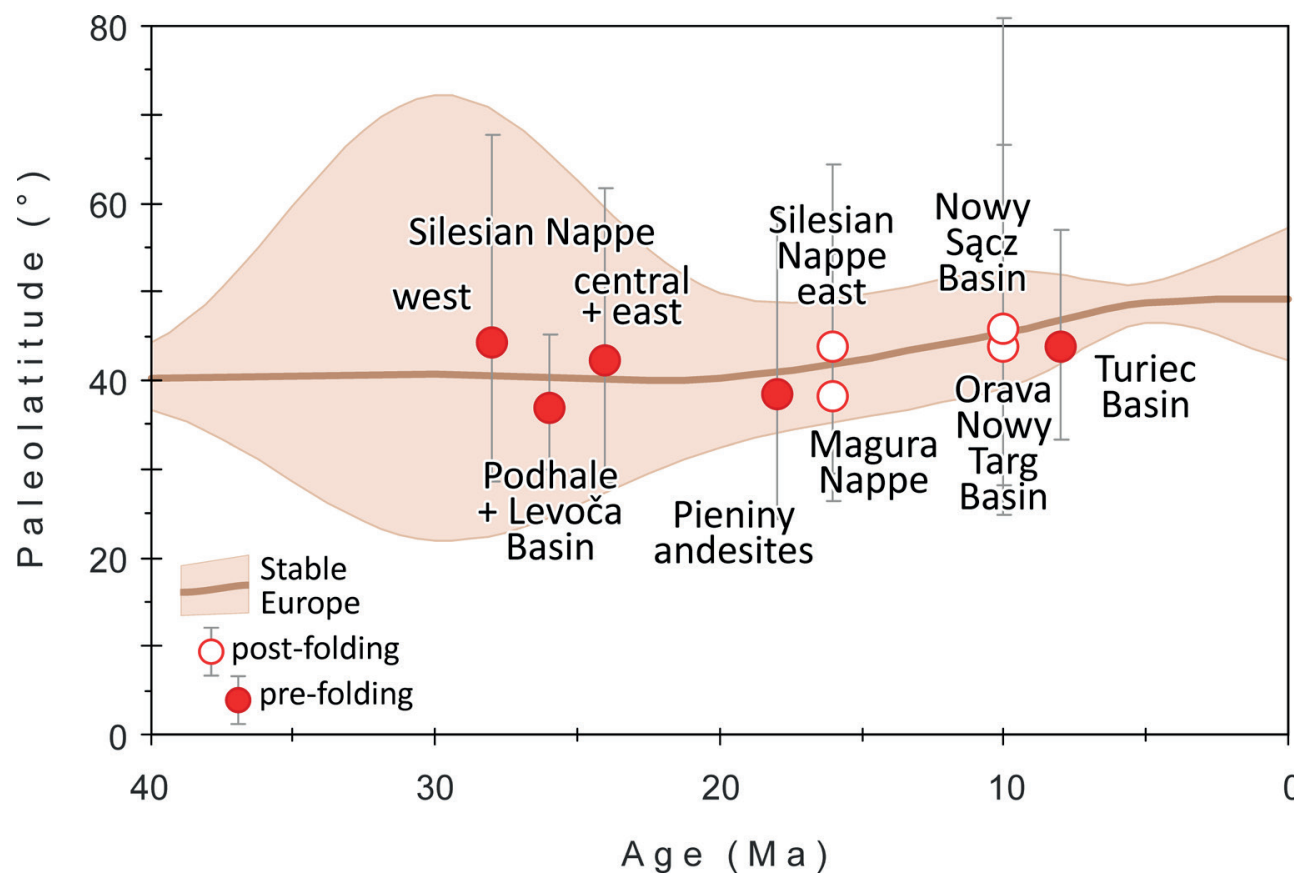

Fig. 6. Paleo-latitudes for the Central and Outer Western Carpathian flysch, the Pieniny andesites, and the sedimentary fills of the intramontane basins (error angles are shown as thin lines) in comparison to expected paleo-latitudes in a stable European framework. The latter is represented by a heavy line showing expected paleolatitudes for $20.45 \mathrm{E}, 49.57 \mathrm{~N}$, calculated from reference poles of Stable Europe for $5 \mathrm{Ma}$ (Panaiotu et al. 2012), $10 \mathrm{Ma}, 20 \mathrm{Ma}$ and $30 \mathrm{Ma}$ (Torsvik et al. 2012). The pale brown area shows the statistical error of stable European paleolatitudes, which are extremely large around $30 \mathrm{Ma}$, due to the scarcity of the paleomagnetic data. The errors for the paleolatitudes were computed with the method by Butler (1992) 
To date, the paleomagnetic results have been discussed from the point of view of large-scale rotation as reflected in the paleomagnetic declinations. It is important, however, that the paleomagnetic inclinations tell us about latitudes at the time of the acquisition of the paleomagnetic signal. As Figure 6 shows, the paleo-latitudes computed from the Western Carpathian paleomagnetic data, as well as the reference paleo-latitudes, have considerable errors and it is therefore hard to estimate the exact distance of the respective areas from the European margin. Nevertheless, the mean paleo-latitudes for the studied Carpathian areas follow closely the reference paleo-latitude curve for stable Europe, thus enhancing the reliability of the paleomagnetic data interpreted in terms of vertical axis rotation.

\section{The tectonic significance of the paleomagnetic results from the Pieniny andesites and from the sediments of the intramontane basins}

\section{Pieniny andesites}

An obvious candidate for a rock unit which is younger than the folding in the Magura Nappe is the Pieniny andesite (Birkenmajer 1986) intruding both the Pieniny Klippen Belt and the southern part of the Magura Nappe (Figs. 1, 7). A pioneering paleomagnetic study, based on alternating field demagnetization (Birkenmajer \& Nairn 1968), concluded that the andesites belonging to two phases of intrusion do not exhibit any rotation.

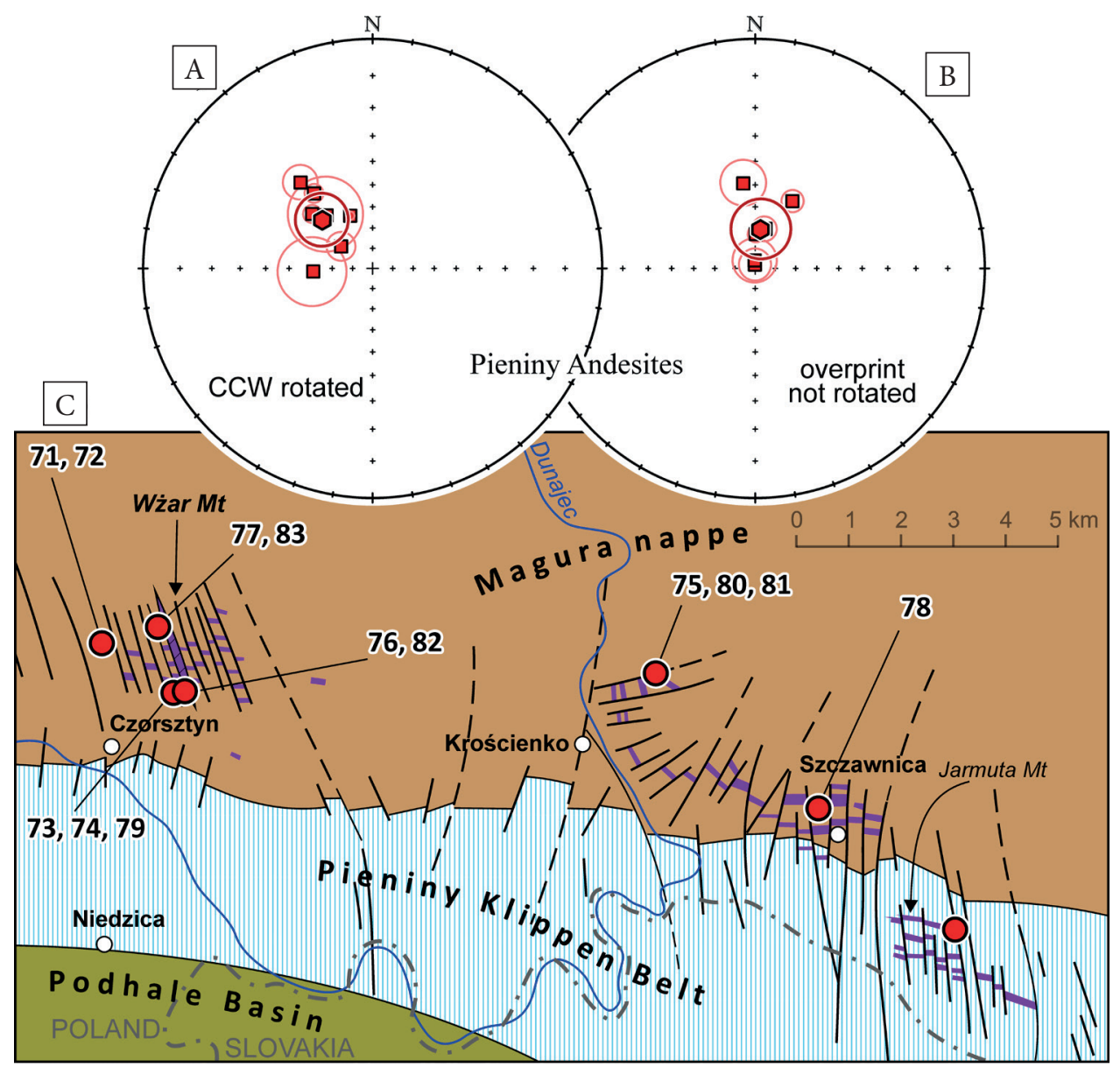

Fig. 7. Pieniny andesites. Paleomagnetic directions with $\alpha_{95}$ on equal angle projection. Figure 7A (Tab. 1, items 71-77): paleomagnetic directions showing CCW rotation. They are interpreted as predating those in Figure $7 B$ (items 78-83). Results tabulated in entries 79-83 exhibit extremely high inclinations, probably "contaminated" due to the superposition of normal and reversed polarity remanences (Márton et al. 2004). Simplified geological map (Fig. 7C) of the sampling area after Birkenmajer (1986), Tokarski \& Świerczewska (1998), and Świerczewska \& Tokarski (1998) 
In a more recent publication (Márton et al. 2004), two paleomagnetic directions were recognized in the laboratory in the course of the more efficient stepwise thermal demagnetization. The one showing no rotation appears as an overprint in most cases, except the Bryjarka quarry in Szczawnica, where the primary remanence seems to have been preserved (Tab. 1, site 78). The other NRM component exhibiting moderate CCW rotation was isolated after the removal of the overprint (Tab. 1, sites 71-77). The timing of this rotation is critical from the point of view of the termination of the large-scale co-ordinated rotation of the Outer Western Carpathian nappes, and in this respect the ages of the andesite intrusions are also critical.

Prior to isotope age studies, the andesite was thought to be of Early Miocene age. However, dating with K-Ar method (Birkenmajer \& Pécskay 1999, 2000) yielded ages spreading from 3.3 to $22.5 \mathrm{Ma}$, with the most reproducible results of 13.5-10.8 Ma. Apatite fission track data (AFT) from the Wżar Mts yielded 18.8 Ma (Anczkiewicz et al. 2005) and 15.8 Ma (Anczkiewicz et al. 2013), respectively. More recent $\mathrm{U}-\mathrm{Pb}$ zircon datings are available from the Jarmuta, Bryjarka and Krupianka streams (11.2-12.1 Ma). However, Anczkiewicz \& Anczkiewicz (2016) do not exclude the possibility that the Wżar intrusions belong to an older phase.

More recent paleomagnetic investigations (Márton et al. 2004) have been concentrated on the Czorsztyn area and particularly on the products of the two phases of intrusions present on the Wżar Mts (Tab. 1, localities 71-74, 76 and 77). In both phases, after the removal of the substantial overprint magnetization by employing the thermal method, CCW rotated magnetizations were isolated (Fig. 7). The andesites near Krościenko behaved similarly and only those in the Bryjarka quarry showeda non-rotated paleomagnetic direction. The above summarized paleomagnetic results, in light of isotope datings, lend themselves to a tentative interpretation concerning the age of about $45^{\circ} \mathrm{CCW}$ rotation, placing it between $18.8 \mathrm{Ma}$ (the oldest AFT age at Wżar Mts, Anczkiewicz et al. 2005) and $11.2 \mathrm{Ma}$ (the youngest U-Pb zircon age at Bryjarka, Anczkiewicz \& Anczkiewicz 2016).

\section{The Orava-Nowy Targ Intramontane Basin}

The Orava-Nowy Targ Intramontane Basin developed at the contact between the Central and Outer Western Carpathians (e.g. Gross et al. 1993, Nagy et al. 1996). It is filled by poorly indurated freshwater Neogene sediments (Fig. 8) up to $1300 \mathrm{~m}$ thick (e.g. Watycha 1976). The youngest rocks underlying the freshwater sequence are the uppermost strata of the Magura Nappe which are around $18 \mathrm{Ma}$ old (Kaczmarek et al. 2016). The freshwater sequence was interpreted as of Late Miocene or Late Miocene-Pliocene age (Tokarski et al. 2016 and references therein). More recently, the uppermost part of the sequence was dated with the U-Pb method as 11.9 Ma at Miętustwo (Wysocka et al. 2018, locality 90 in Fig. 8). It is discordantly covered by Quaternary fluvial sediments. The paleomagnetic directions of post-tilting age from six localities (Tab. 1, localities 84-89) suggest about $30^{\circ} \mathrm{CCW}$ rotation (Fig. 8). This rotation was interpreted as connected to the sinistral strike-slip movement along the Mur-Žilina Fault Zone (Fig. 1, Tokarski et al. 2016, cf. Ludwiniak et al. 2019), which was active mainly between 17 and $8 \mathrm{Ma}$ and then again from the Middle Pleistocene on (Decker et al. 2005; see also Baumgart-Kotarba et al. 2004). According to the paleomagnetic results, the CCW rotation must be older than 11.9 Ma, since the paleo-declination for localities 90 and 91 (Tab. 1) are close to the north, i.e. postdates the rotation.

\section{Nowy Sacz Intramontane Basin}

The Nowy Sącz Intramontane Basin is a piggy-back basin opened up within the Magura Nappe (e.g. Oszczypko 1973). Its Neogene to Quarternary sedimentary fill rests upon folded and eroded sediments of the Magura Nappe (Oszczypko 1973; Oszczypko et al. 1999). In the Magura Nappe, where a large CCW rotation was observed (Márton et al. 2009a), the youngest strata are late Oligocene-Miocene age (around $23 \mathrm{Ma}$, Oszczypko-Clowes et al. 2018). The oldest part of the poorly indurated the Nowy Sącz Basin (Fig. 9) fill is considered to be about $14 \mathrm{Ma}$, whereas its youngest part is around $12 \mathrm{Ma}$ (Oszczypko-Clowes et al. 2009). The Neogene strata are open folded and the deformation must have post-dated $12 \mathrm{Ma}$. 


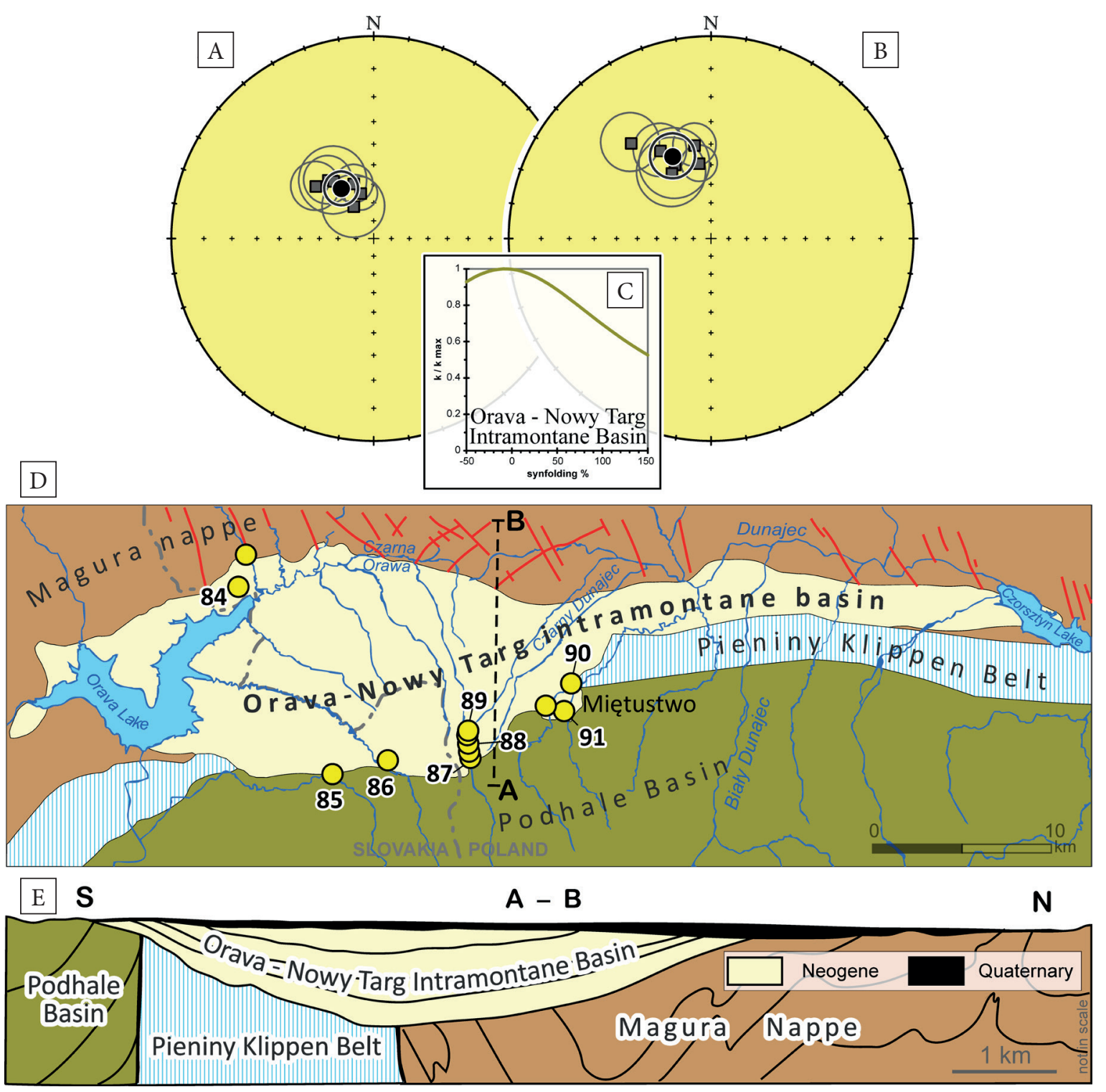

Fig. 8. Orava-Nowy Targ Intramontane Basin. Paleomagnetic directions (Tab. 1, items 84-89) with $\alpha_{95}$ on equal angle projection before (Fig. 8A) and after (Fig. 8B) tilt corrections accompanied by syn-folding diagram (Fig. 8C). Simplified geological map (Fig. 8D) and simplified cross section (Fig. 8E) of the basin after Watycha (1977a, 1977b), Lexa et al. (2000), Pomianowski (2003) and Tokarski et al. (2016)

The paleomagnetic results represent the bottom part of the basin filling. Some samples (in 2000) were collected from an active brickyard (Tab. 1, locality 97), others from natural outcrops (Tab. 1,98102) along the Kamienica River (Fig. 9D).

As Figure 9C documents, the tilt test is indeterminate, with considerably less scatter before than after tilt corrections. The exact age of the acquisition of the magnetizations is not critical from the viewpoint of tectonic interpretation, since the overall-mean paleomagnetic declinations are very similar both before and after tilt corrections.
A remarkable aspect of the results is the reversed polarity magnetization at all the studied localities. This rules out the possibility of recent remagnetization and at the same time, suggests that the magnetizations at the different localities were most likely acquired within the same polarity zone.

In summary, the results document that the Neogene sequence filling the Nowy Sącz Intramontane Basin did not experience any rotation after the magnetization of the Miocene sediments. However, it is impossible to pinpoint the age of the acquisition of remanence for two reasons. 

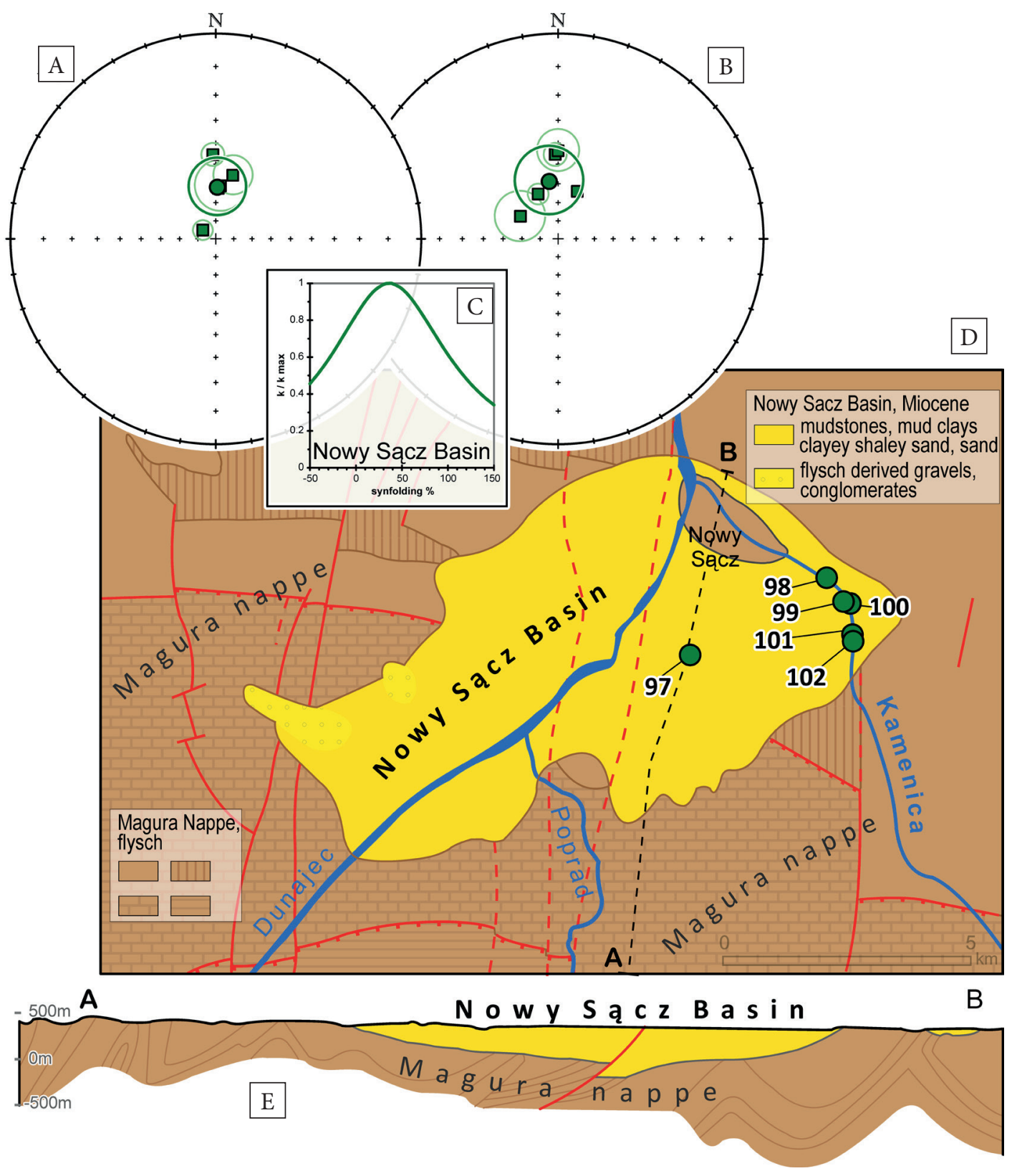

Fig. 9. Nowy Sacz Intramontane Basin. Paleomagnetic locality mean directions with $\alpha_{95}$ on equal angle projection before (Fig. 9A) and after (Fig. 9B) tilt correction, accompanied by syn-tilting diagram (Fig. 9C). The locality mean paleomagnetic direction for locality 98 is without $\alpha_{95}$ as it was determined from a great circle defined by the individual specimens (pole of the circle $D=143.0^{\circ}, I=21.9^{\circ}, \alpha_{95}=7.6^{\circ}$, length $\left.=153^{\circ}\right)$ as the vector, which was at the minimum distance from the cluster of the locality mean directions for localities 97 and 99-101 (method by McFadden \& McElhinney 1988). Simplified geological map (Fig. 9D) and simplified cross section (Fig. 9E) of the basin after Oszczypko et al. (1999) and Oszczypko-Clowes et al. (2009)

One is the indeterminate tilt tests and the other is the uncertain age of the tectonic process responsible for the tilting of the strata. Therefore, the results represent a loose control on the termination of the large-scale rotation of the Western Carpathians, permitting it to be placed to any time period after $14 \mathrm{Ma}$.

\section{Turiec Intramontane Basin}

The Turiec Intramontane Basin, a westward dipping half-graben (Kováč et al. 2011), is the largest one in the Central Western Carpathians (Fig. 1) which existed during the Middle and Late Miocene (e.g. Hók et al. 1998, Kováč et al. 2011). It is surrounded by uplifted Paleozoic crystalline 
basement with its Mesozoic sedimentary cover overlain by nappe units composed mostly of carbonate rocks (Fig. 10). Erosive remnants of Central Carpathian Paleogene Basin are found in the northeast margin of the basin (Gašparík et al. 1995). The sedimentary fill of the basin, reaching up to $1250 \mathrm{~m}$, was episodically connected with the Pannonian Basin (Kováč \& Hók 1993, Pipík et al. 2012).

One of the time constraints on the otherwise loose age control on the sedimentary fill of the basin is a rhyolite volcanic complex (radiometric age $12.4 \pm 2.2$ to $10.7 \pm 0.3 \mathrm{Ma}$, Gašparík et al. 1974, Konečný et al. 1983). This is situated in the lower part of the sedimentary sequence, well below the lake sediments. The subsidence of the Turiec Basin reached a maximum when connections towards the Pannonian Basin were cut by the rapid uplift of the surrounding mountains and the arising strato-volcanoes (Králiková et al. 2014) and when deepwater lacustrine sediments and littoral mudstone and sand intercalated with bodies of freshwater limestone and coal seams were deposited. These sediments were the subjects of a paleomagnetic study. Correlation to the world-wide polarity time scale combined with the development of the rich Ostracode population assigned an age of 8.1-7.7 Ma (Márton et al. in prep.) to the lake sediments studied for paleomagnetism (Tab. 1, localities 92-96). These sediments have magnetizations of pre-tilting age (Fig. 10D) and show practically no rotation with respect to the present orientation (Márton et al. in prep.). This result is the most reliable evidence to date that the large-scale rotation of the Central and Outer Western Carpathians was definitely over around $8 \mathrm{Ma}$.

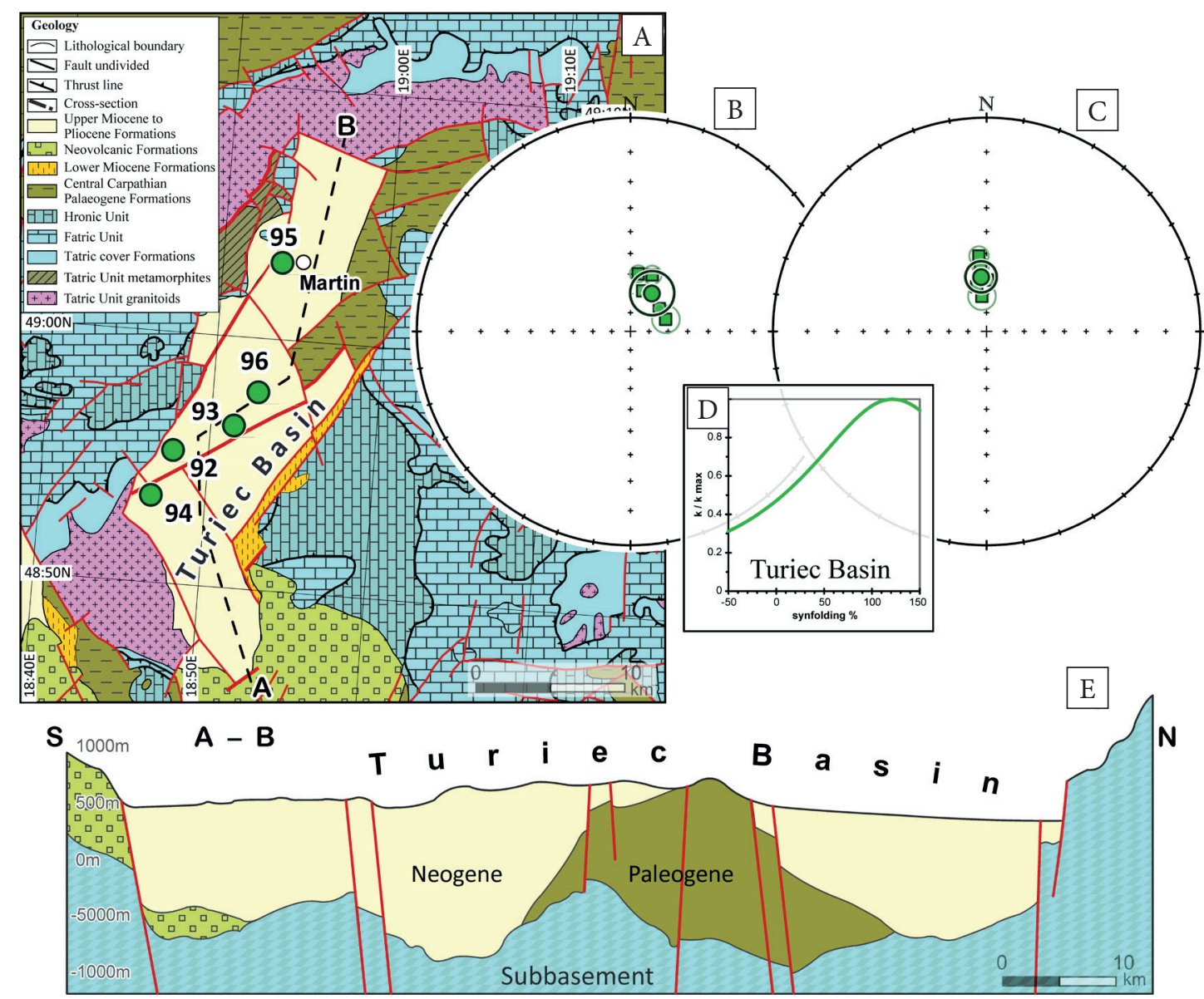

Fig. 10. Turiec Intramontane Basin. Paleomagnetic directions (Tab. 1, items 79-83) with $\alpha_{95}$ on equal angle projection before (Fig. 10B) and after (Fig. 10C) tilt corrections accompanied by syn-folding diagram (Fig. 10D). Simplified geological map (Fig. 10A) and simplified cross section (Fig. 10E) of the basin after Zbořil et al. (1985), Gašparík et al. (1995), Bezák et al. (2004), Kováč et al. (2011), Pipík et al. (2012) and Králiková et al. (2014) 


\section{CONCLUSIONS}

The paleomagnetic results from the Silesian, the Dukla and the Magura nappes outline the following scenario. The Paleogene-Lower Miocene flysch deposited in the different basins of the Outer Western Carpathians acquired remanent magnetization before folding. This remanence was preserved at most localities of the Silesian Nappe, while at some others and at all the studied localities in the Magura Nappe, the flysch was remagnetized after folding, most probably in the Miocene after the deposition of the youngest members of the Krosno beds in the Silesian Nappe (Tab. 1, locality 22). The magnetizations of pre-folding as well as of post-folding ages suggest about $50^{\circ}$ general CCW rotation of the nappes, with respect to north, which is about $60^{\circ}$ with respect to stable Europe (Fig. 11). The implication is that the general orientation of the Magura, the Dukla and Silesian nappes during folding was NW-SE, making an angle of about $50^{\circ}$ with their present orientation. Concerning the pre-rotation orientation, it is comparable with the reconstruction e.g. by Rakús
(1998), Oszczypko (2006) for the Maastrichtian, or by Picha et al. (2006) for the Oligocene. The paleomagnetic data constrain the necessary CCW rotation for the emplacement of the nappe systems to the Miocene, as also suggested by the reconstruction of Kováč et al. (2016) for 26-22 Ma.

Some of the Pieniny andesite intrusions, mostly in the Wżar Mts area, have two components of the natural remanent magnetization. The more stable one, interpreted as primary, suggest about $45^{\circ} \mathrm{CCW}$ vertical axis rotation with respect to north (Fig. 11). The less stable components indicate no vertical axis rotation, similarly to a single component paleomagnetic direction characterizing the andesite from the Bryjarka quarry in Szczawnica. The oldest apatite fission track age at Wżar is around 18.8 Ma (Anczkiewicz et al. 2005) and the youngest zircon $\mathrm{U}-\mathrm{Pb}$ age at Bryjarka is around 11.2 Ma (Anczkiewicz \& Anczkiewicz 2016). Relying on these isotope ages, and the paleomagnetic results from the Pieniny andesites, the large scale CCW vertical axis rotation of the Outer Western Carpathian nappes can be tentatively placed between 18 and 11 Ma.

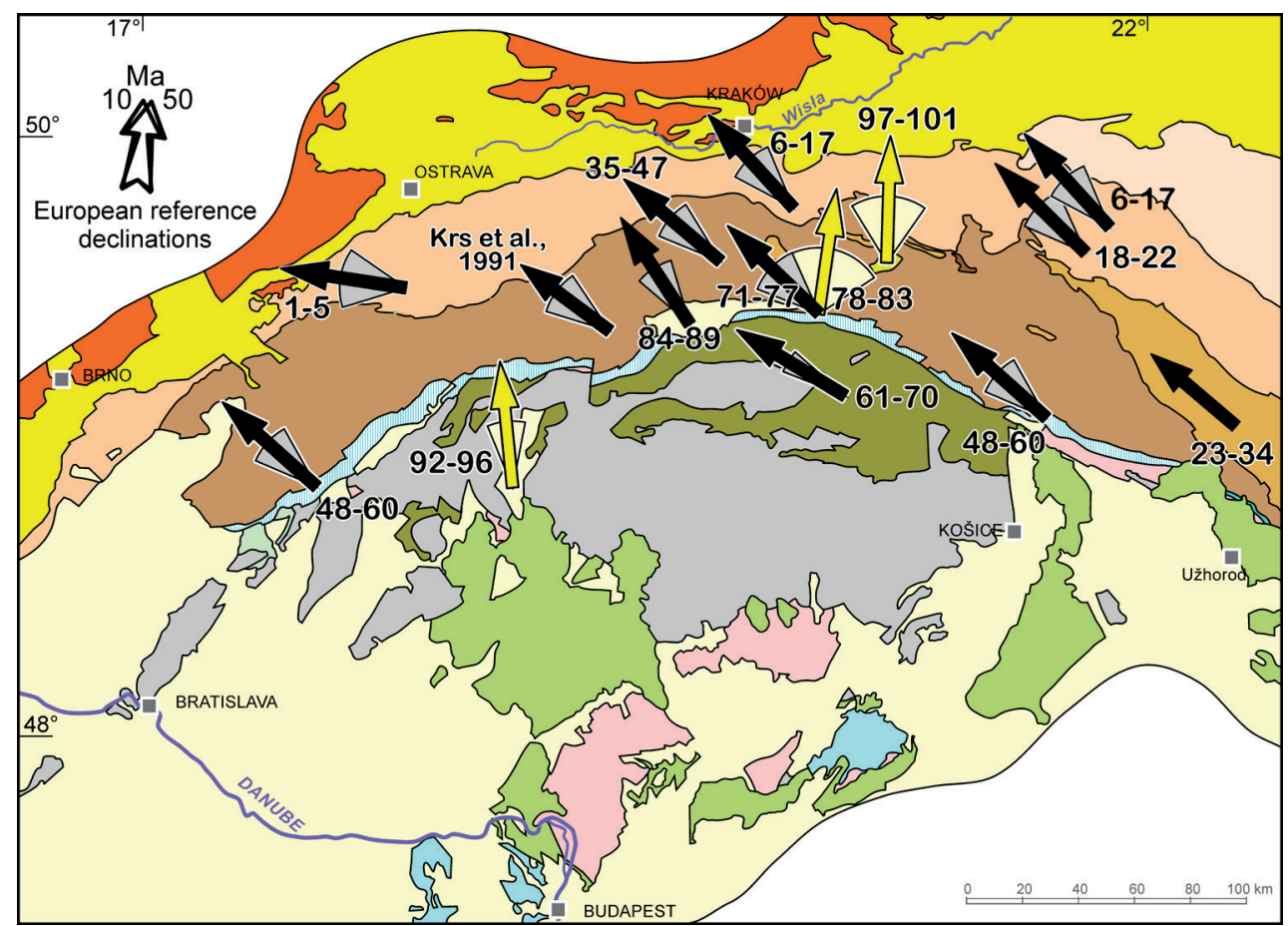

Fig. 11. A simplified geological map of the study area with the overall-mean paleomagnetic declinations (with envelopes showing $\Delta D$, the statistical error of declination, Butler 1992) characterizing the tectonostratigraphic units studied. Numbers refer to Table 1. Please note that the arrows close to the western and eastern ends of the Pieniny Klippen Belt, respectively, are valid for the Belt between the two arrows 
The above age estimation is corroborated, or at least not contradicted, by the paleomagnetic results from the intramontane basins. The most reliable evidence for the absence of vertical axis rotation of the Western Carpathians is from the Turiec Intramontane Basin, where the magnetizations of the sediments are of pre-tilting age. The results suggest that the rotation was definitely over by around $8 \mathrm{Ma}$.

The CCW rotation observed for the sedimentary fill of the Orava-Nowy Targ Intramontane Basin predates 11.9 Ma, thus it would fit the time period outlined by the Pieniny andesites. However, this rotation was not connected to the CCW rotation related to the emplacement of the Outer Western Carpathian nappes over the sediments of the Carpathian Foredeep, but rather to the strike-slip displacement along the left lateral shear zone of the Mur-Žilina Fault Zone (Tokarski et al. 2016).

The results from the Nowy Sącz Intramontane Basin are in harmony with those for the Turiec Intramontane Basin, although their power to constrain the termination of the rotation is less due to the post-tilting age of the magnetization.

The author is indebted to the co-authors of the several papers published and cited about the paleomagnetism of the overviewed area over the last two decades. My special thanks are due to Antek Tokarski, who has been the best co-operation partner in most of the investigations covered by this paper: His field experience and excellent organization of the sampling campaigns in Poland, as well as his critical assessment of the available geological information, has been instrumental in collecting suitable samples in the field and the interpretation of the paleomagnetic results. Constructive reviews by Jozef Madzin and three anonymous reviewers as well as some editorial comments by Anna Świerczewska greatly helped to improve the manuscript. Financial support from Project NKFIH K-128625 is gratefully acknowledged.

\section{REFERENCES}

Aleksandrowski P., 1985. Tektonika regionu babiogórskiego: interferencja zachodnio- i wschodniokarpackich kierunków fałdowych [A structure of the Mount Babia Góra region (magura nappe, Western outer Carpoathians): an inference of West and East Carpathians fold trends]. Annales Societatis Geologorum Poloniae, 55, 375-422.
Anczkiewicz A.A. \& Anczkiewicz R., 2016. U-Pb zircon geochronology and anomalous $\mathrm{Sr}-\mathrm{Nd}$-Hf isotope systematics of late orogenic andesites: Pieniny Klippen Belt, Western Carpathians, South Poland. Chemical Geology, 427, 1-16. https://doi.org/10.1016/j.chemgeo.2016.02.004.

Anczkiewicz A.A., Zattin M. \& Środoń J., 2005. Cenozoic uplift of the Tatras and Podhale Basin from the perspective of the apatite fission track analyses. Polskie Towarzystwo Mineralogiczne. Prace specjalne, 25, 261-264.

Anczkiewicz A.A., Środoń J. \& Zattin M., 2013. Thermal history of the Podhale Basin in the Internal Western Carpathians from the perspective of apatite fission track analyses. Geologica Carpathica, 64, 141-151. https://doi. org/10.2478/geoca-2013-0010.

Baumgart-Kotarba M., Marcak H. \& Márton E., 2004. Rotation along the transverse transforming Orava strike-slip fault: based on geomorphological, geophysical and paleomagnetic data (Western Carpathians). Geologica Carpathica, 55, 3, 219-226.

Bezák V., Broska I., Ivanička J., Reichwalder P., Vozár J., Polák M., Havrila M., Mello J., Biely A., Plašienka D., Potfaj M., Konečný V., Lexa J., Kaličiak M., Žec B., Vass D., Elečko M., Janočko J., Pereszlényi M., Marko F., Maglay J. \& Pristaš J., 2004. Tektonická mapa Slovenskej republiky. Ministerstvo životného prostredia Slovenskej republik [Tectonic Map of Slovak Republic. Ministry of Environment of the Slovak Republic]. Štátny geologický ústav Dionýza Štúra, Bratislava.

Birkenmajer K., 1986. Stages of structural evolution of the Pieniny Klippen Belt, Carpathians. Studia Geologica Polonica, 88, 7-32.

Birkenmajer K. \& Nairn A., 1968. Paleomagnetic studies of Polish rocks. III. Neogene igneous rocks of the Pieniny Mountains, Carpathians. Annales Societatis Geologorum Poloniae, 38, 475-489.

Birkenmajer K. \& Pécskay Z., 1999. K-Ar dating of the Miocene andesite intrusions, Pieniny Mts, West Carpathians, Poland. Bulletin of the Polish Academy of Sciences. Earth Sciences, 47, 155-169.

Birkenmajer K. \& Pécskay Z., 2000. K-Ar dating of the Miocene andesite intrusions, Pieniny Mts, West Carpathians, Poland: a supplement. Studia Geologica Polonica, 117, 7-25.

Butler R.F., 1992. Paleomagnetism: Magnetic Domains to Geologic Terranes. Blackwell Scientific Publications.

Decker K., Peresson H. \& Hinsch R., 2005. Active tectonics and Quaternary basin formation along the Vienna Basin Transform fault. Quaternary Science Reviews, 24, 305-320.

Enkin R.J., 2003a. The direction-correction tilt test: an all-purpose tilt/fold test for paleomagnetic studies. Earth and Planetary Science Letters, 212, 151-166. https://doi. org/10.1016/S0012-821X(03)00238-3.

Enkin R.J., 2003b. PMGSC Paleomagnetism Data Analysis, v 4.2. Geological Survey of Canada, Sidney.

Filo I. \& Siráňová Z., 1996. Tomášovské vrstvy - nova litostratigrafická jednotka podtatranskej skupiny [The Tomášovce Member - a new lithostratigraphic unit of the Subtatric Group]. Geologicke práce, správy, 102, 41-49.

Fisher R.A., 1953. Dispersion on a sphere. Proceedings of the Royal Society London, 217, 295-305. 
Fodor L., Francu J., Krejčí O. \& Stráník Z., 1995. Palaeogeographic and Tectonic Evolution of the Carpathian Flysch Belt of Southern Moravia (Czech Republic). Special Publications, Geological Society of Greece, 4/1.

Gašparík J., Brestenská E., Forgáč J., Franko O., Hajósová M., Hanáček J., Marková M., Matkulčík E., Planderová E. \& Sitár V., 1974. Štruktúrny vrt GHŠ-1 (HornáŠtubřa) [Structural borehole GHŠ-1 (Horná Štubňa)]. Regionálna geológia, 3, Štátny geologický ústav Dionýza Štúra, Bratislava.

Gašparík J., Halouzka R., Miko O., Gorek J., Rakús M., Bujnovský A., Lexa J., Panáček A., Samuel O., Gašparíková V., Planderová E., Snopková P., Fendek M., Hanáček J., Modlitba I., Klukanová A., Žáková E., Horniš J. \& Ondrejičková A., 1995. Výsvetlivky ku geologickej mape Turčianskej kotliny 1:50 000 [Explanations to geological map 1:50 000 of the Turiec Depression]. Štátny geologický ústav Dionýza Štúra, Bratislava.

Gedl P., 1998. Wiek fliszu podhalańskiego na podstawie dinocyst. Przeglad Geologiczny, 46, 45-46.

Golonka J., Krobicki M., Waśkowska A., Cieszkowski M. \& Ślączka A., 2015. Olistostromes of the Pieniny Klippen Belt, Northern Carpathians. Geological Magazine, 152, 269-286.

Golonka J., Krobicki M. \& Waśkowska A., 2018. The Pieniny Klippen Belt in Poland. Geology, Geophysics \& Environment, 44, 111-125.

Golonka J., Pietsch K., Marzec P., Kasperska M., Dec J., Cichostępski K. \& Lasocki S., 2019. Deep structure of the Pieniny Klippen Belt in Poland. Swiss Journal of Geosciences, 112, 475-506.

Gross P., Köhler E., Haško J., Halouzka R., Mello J. \& Nagy A., 1993. Geológia juźnej a východnej Oravy [Geology of southern and eastern Orava]. Štátny geologický ústav Dionýza Štúra, Bratislava.

Hók J., Kováč M., Rakús M., Kováč P., Nagy A., Kováčová-Slamková M., Sitár V. \& Šujan M., 1998. Geologic and tectonic evolution of the Turiec depression in the Neogene. Slovak Geological Magazine, 4, 165-176.

Janočko J., 2002. Centrálnokarpatský paleogénny bazén vývoj a depozičné systémy [Central-Carpathian Paleogene basin - evolution and depositional systems]. Mineralia Slovaca, 34, 159-180.

Janočko J. \& Jacko S., 1998. Marginal and deep-sea deposits of Central-Carpathian Paleogene Basin, Spišská Magura region, Slovakia. Implication for basin history. Slovak Geological Magazine, 4, 4, 281-292.

Janočko J., Hamršmíd B., Jacko S. \& Siráňová Z., 1998. Suprafan and chanell-and-levee deposits near Tichý Potok, Levoča Mts.; Central-Carpathian Paleogene Basin, Slovakia. Slovak Geological Magazine, 4, 1, 3-15.

Kaczmarek A., Oszczypko-Clowes M. \& Cieszkowski M., 2016. Early Miocene age of the Stare Bystre Formation based on calcareous nannofossils (Magura Nappe, Outer Carpathians, Poland). Geological Quarterly, 60, 2, 341-354.

Kent J.T., Briden J.C. \& Mardia K.V., 1983. Linear and planar structure in order multivariate data as applied to progressive demagnetization of paleomagnetic remanence. Geophysical Journal of the Royal Astronomical Society, $75,593-621$

Kirschvink J.L., 1980. The least-squares line and plane and the analysis of paleomagnetic data. Geophysical Journal of the Royal Astronomical Society, 62, 699-718.
Kiss D., Márton E. \& Tokarski A.K., 2016. An integrated paleomagnetic and magnetic anisotropy study of the Oligocene flysch from the Dukla nappe, Outer Western Carpathians, Poland. Geologica Carpathica, 57, 6, 595-605.

Konečný V., Lexa J. \& Planderová E., 1983. Stratigrafické členenie neovulkanitov stredného Slovenska [Stratigraphy of the Central Slovakia Volcanic Field]. Západné Karpaty, séria Geológia, 9, Štátny geologický ústav Dionýza Štúra, Bratislava.

Koráb T., Krs M., Krsová M. \& Pagáč P., 1981. Paleomagnetic Investigations of Albian(?)-Paleocene to Lower Eocene Sediments from the Dukla Unit, East Slovakian Flysch, Czechoslovakia. Západné Karpaty, séria Geológia, 7, Štátny geologický ústav Dionýza Štúra, Bratislava, 127-149.

Kováč P. \& Hók J. 1993. The Central Slovakia fault System field evidence of a strike slip. Geologica Carpathica, 44, 3, 155-159.

Kováč M., Hók J., Minár J., Vojtko R., Bielik M., Pipík R., Rakús M., Král J., Šujan M. \& Králiková S., 2011. Neogene and Quaternary development of the Turiec Basin and landscape in its catchment. A tentative mass balance model. Geologica Carpathica, 62, 361-379.

Kováč M., Plašienka D., Soták J., Vojtko R., Oszczypko N., Less G., Ćosović V., Fügenschuh B. \& Králiková S., 2016. Paleogene palaeogeography and basin evolution of the Western Carpathians, Northern Pannonian domain and adjoining areas. Global and Planetary Change, 140, 9-27.

Kováč M., Márton E., Oszczypko N., Vojtko R., Hók J., Králiková S., Plašienka D., Klučiar T., Hudáčková N. \& Oszczypko-Clowes M., 2017. Neogene palaeogeography and basin evolution of the Western Carpathians, Northern Pannonian domain and adjoining areas. Global Planetary Change, 155, 133-154.

Kováč M., Márton E., Klučiar T. \& Vojtko R., 2018. Miocene basin opening in relation to the north-eastward tectonic extrusion of the ALCAPA Mega-Unit. Geologica Carpathica, 69, 3, 254-263.

Králiková S., Vojtko R., Andriessen P., Kováč M., Fügenschuh B., Hók J. \& Minár J., 2014. Late Cretaceous-Cenozoic thermal evolution of the northern part of the Central Western Carpathians (Slovakia): revealed by zircon and apatite fission track thermochronology. Tectonophysics, 615-616, 142-153.

Krs M., Krsová M., Chvojka R. \& Potfaj M., 1991. Paleomagnetic investigations of the flysch belt in the Orava region, Magura unit, Czechoslovak Western Carpathians. Geologické práce, Správy, 92, 135-151.

Lexa J., Bezák V., Elečko M., Mello J., Polák M., Potfaj M. \& Vozár J. (eds.), 2000. Geologická mapa Západných Karpát a prilahlých území 1:500 000. Ministerstvo životného prostredia Slovenskej republiky [Geological map of Western Carpathians and adjacent areas 1: 500,000. Ministry of Environment of Slovak Republic]. Štátny geologický ústav Dionýza Štúra, Bratislava.

Łoziński M., Wysocka A. \& Ludwiniak M., 2015. Neogene terrestrial sedimentary environments of the Orava-Nowy Targ Basin: a case study of the Oravica River section near Čimhová, Slovakia. Geological Quarterly, 59, 21-34.

Ludwiniak M., Śmigielski M., Kowalczyk S., Łoziński M., Czarniecka U. \& Lewińska L., 2019. The intramontane Orava Basin - evidence of large-scale Miocene to Quaternary sinistral wrenching in the Alpine-Carpathian-Pannonian area. Acta Geologica Polonica, 69, 3, 339-386. https://doi.org/10.24425/agp.2019.126449. 
Márton E., Mastella L. \& Tokarski A.K., 1999. Large counterclockwise rotation of the Inner West Carpathian Paleogene Flysch - evidence from paleomagnetic investigation of the Podhale Flysch (Poland). Physics and Chemistry of the Earth, A24, 8, 645-649.

Márton E., Tokarski A.K. \& Halász D., 2004. Late Miocene counterclockwise rotation of the Pieniny andesites at the contact of the Inner and Outer West Carpathians. Geologica Carpathica, 55, 5, 411-419.

Márton E., Rauch-Włodarska M., Krejčí O., Tokarski A.K. \& Bubík M., 2009a. An integrated palaeomagnetic and AMS study of the Tertiary flysch from the Outer Western Carpathians. Geophysical Journal International, 177, 925-940. https://doi.org/10.1111/j.1365-246X2009.04104.x.

Márton E., Jeleńska M., Tokarski A.K., Soták J., Kováč M. \& Spišiak J., 2009b. Current-independent paleomagnetic declinations in flysch basins: a case study from the Inner Carpathians. Geodinamica Acta, 22, 1-3, 73-82. https:// doi.org/10.3166/ga.22.73-82.

Márton E., Tokarski A.K., Krejčí O., Rauch M., Olszewska B., Tomanová Petrová P. \& Wójcik A., 2011. "Non European" paleomagnetic directions from the Carpathian Foredeep at the southern margin of the European plate. Terra Nova, 23, 134-144. https://doi.org/10.1111/ j.1365-3121.2011.00993.x.

Márton E., Grabowski J., Plašienka D., Túnyi I., Krobicki M., Haas J. \& Pethe M., 2013. New paleomagnetic results from the Upper Cretaceous red marls of the Pieniny Klippen Belt, Western Carpathians: evidence for general CCW rotation and implications for the origin of the structural arc formation. Tectonophysics, 592, 1-13. https://doi.org/10.1016/j.tecto.2013.01.027.

Márton E., Grabowski J., Tokarski A.K. \& Túnyi I., 2016. Palaeomagnetic results from the fold and thrust belt of the Western Carpathians: an overview. [in:] Pueyo E.L., Cifelli F., Sussman A.J. \& Oliva-Urcia B. (eds.), Palaeomagnetism in Fold and Thrust Belts: New Perspectives, Geological Society, London, Special Publications, 425, 7-36. https://doi.org/10.1144/SP425.1.

Márton E., Zampieri D., Ćosović V., Moro A. \& Drobne K., 2017. Apparent Polar Wander Path for Adria extended by new Jurassic paleomagnetic results from its stable core: tectonic implications. Tectonophysics, 700-701, 1-18. https://doi.org/j.tecto.2017.02.004.

Márton E., Pipik R., Starek D., Šurka J., Kovács E., Vidhya M., Świerczewska A., Tokarski A.K., Vojtko R. \& KralikováS., in prep. Enhancing the reliability of the magnetostratigrahic age assignment of azimuthally non-oriented drill cores by the integrated application of palaeomagnetic analysis, field tests, AMS, and the gradual evolution of the endemic fauna as documented on the Late Miocene limnic deposits of the Turiec Basin (Central Western Carpathians).

Marzec P., Golonka J., Pietsch K., Kasperska M., Dec J., Cichostępski K. \& Lasocki S., 2020. Seismic imaging of mélanges - Pieniny Klippen Belt case study. Journal of the Geological Society, 177, 629-646.

McFadden P.L. \& McElhinney M.W., 1988. The combined analysis of remagnetization circles and direct observations in paleomagnetism. Earth and Planetary Science Letters, 87, 161-172.
McFadden P.L. \& McElhinney M.W., 1990. Classification of the reversal test in palaeomagnetism. Geophysical Journal International, 103, 725-729.

Nagy A., Vass D., Petrik F. \& Pereszlényi M., 1996. Tectogenesis of the Orava Depression in the light of latest biostratigraphic investigations and organic matter alteration studies. Slovak Geological Magazine, 1, 49-58.

Nemčok M., Krzywiec P., Wojtaszek M., Ludhová L., Klecker R.A., Sercombe W.J. \& Coward M.P., 2006. Tertiary development of the Polish and eastern Slovak parts of the Carpathian accretionary wedge: insights from balanced cross-sections. Geologica Carpathica, 57, 5, 355-370.

Oszczypko N., 1973. Budowa geologiczna Kotliny Sądeckiej [The geology of the Nowy Sącz Basin, Middle Carpathians]. Biuletyn Instytutu Geologicznego, 271, 101-197.

Oszczypko N., 2006. Late Jurassic-Miocene evolution of the Outer Carpathian fold-and thrust belt and its foredeep basin (Western Carpathians, Poland). Geological Quarterly, 50, 169-194.

Oszczypko N. \& Oszczypko-Clowes M., 2009. Stages in the Magura Basin. a case study of the Polish sector (Western Carpathians). Geodinamica Acta, 22, 83-100.

Oszczypko N., Andreyeva-Grigorovich A.S., Malata E. \& Oszczypko-Clowes M.A., 1999. The Lower Miocene deposits of the Rača subunit near Nowy Sącz (Magura nappe, Polish Outer Carpathians). Geologica Carpathica, 50, 416-433.

Oszczypko-Clowes M., Oszczypko N. \& Wójcik A., 2009. New data on the late Badenian-Sarmatian deposits of the Nowy Sącz Basin (Magura Nappe, Polish Outer Carpathians) and their palaeogeographicacal implications. Geological Quarterly, 53, 273-292.

Oszczypko-Clowes M., Oszczypko N., Piecuch A., Soták J. \& Boratyn J., 2018. The Early Miocene residual flysch basin at the front of the Central Western Carpathians and its palaeogeographic implications (Magura Nappe, Poland). Geological Quaterly, 62, 597-619.

Panaiotu C.G., Visan M., Tugui A., Seghedi I. \& Panaiotu A.G., 2012. Palaeomagnetism of the South Harghita volcanic rocks of the East Carpathians: implications for tectonic rotations and palaeosecular variation in the past 5 Ma. Geophysical Journal International, 189, 369-382. https://doi.org/10.1111/j.1365-246X.2012.05394.x.

Picha F.J., Stráník Z. \& Krejčí O., 2006. Geology and hydrocarbon resources of the Outer Western Carpathians and their foreland, Czech Republic. [in:] Golonka J. \& Picha F.J. (eds.), The Carpathians and their foreland: Geology and hydrocarbon resources, AAPG Memoir, 84, 49-175.

Pipík R., Bodergat A-M., Briot D., Kováč M., KrálJ. \& Zielinski G., 2012. Physical and biological properties of the late Miocene, long-lived Turiec Basin, Western Carpathians (Slovakia) and its paleobiotopes. Journal of Paleolimnology, 47, 233-249. https://doi.org/10.1007/s10933-0119573-2.

Plašienka D., 2012. Early stages of structural evolution of the Carpathian Klippen Belt (Slovakian Pieniny sector). Mineralia Slovaca, 44, 1, 1-16.

Plašienka D., 2018. Continuity and Episodicity in the Early Alpine Tectonic Evolution of the Western Carpathians: How Large-Scale Processes Are Expressed by the Orogenic Architecture and Rock Record Data. Tectonics, 37, 2029-2079. https://doi.org/10.1029/2017TC004779. 
Pomianowski P., 2003. Tektonika Kotliny Orawsko-Nowotarskiej - wyniki kompleksowej analizy danych grawimetrycznych i geoelektrycznych [Tectonics of the Orava-Nowy Targ Basin - results of the combined analysis of the gravity and geoelectrical data]. Przeglad Geologiczny, 51, 498-506.

Rakús M., 1998. Principles and methods applied to create geodynamic model. [in:] Rakús M. (ed.), Geodynamic development of the Western Carpathians, Geological Survey of Slovak Republic, Bratislava, 9-15.

Scholger R. \& Stingl K., 2004. New paleomagnetic results from the middle Miocene (Karpatian and Badenian) in Northern Austria. Geologica Carpathica, 55, 199-206.

Siemińska A., Starzec K., Waśkowska A. \& Wendorff M. 2020. Sedimentary and diapiric mélanges in the Skrzydlna area (Outer Carpathians of Poland) as indicators of basinal and structural evolution. Journal of the Geological Society of London, 177, 600-618.

Soták J., Pereszlenyi M., Marschalko R., Milička J. \& Starek D., 2001. Sedimentology and hydrocarbon habitat of the submarine-fan deposits of the Central Carpathian Paleogene Basin(NE Slovakia). Marine Petroleum Geology, 18, 87-114.

Świerczewska A. \& Tokarski A.K., 1998. Deformation bands and the history of folding in the Magura nappe, Western Outer Carpathians (Poland). Tectonophysics, 297, 73-90.

Tokarski A.K. \& Świerczewska A., 1998. History of folding in the Magura Nappe, Outer Carpathians, Poland. [in:] Rossmanith H.-P. (ed.), Mechanicsof Faulted and Jointed Rock, Balkema, 125-130.

Tokarski A.K., Świerczewska A., Zuchiewicz W., Starek D. \& Fodor L., 2012. Quaternary exhumation of the Carpathians: a record from the Orava-Nowy Targ Intramontane Basin, Western Carpathians (Poland and Slovakia). Geologica Carpathica, 63, 257-266.
Tokarski A.K., Márton E., Swierczewska A., Fheed A., Zasadni J. \& Kukulak J., 2016. Neotectonic rotations in the Orava-Nowy Targ Intramontane Basin (Western Carpathians): An integrated palaeomagnetic and fractured clasts study. Tectonophysics, 685, 35-43. https://doi.org/ 10.1016/j.tecto.2016.07.013.

Torsvik T.H., van der Voo R., Preeden U., Mac Niocaill C., Steinberger B., Doubrovine P.V., van Hinsbergen D.J.J., Domeier M., Gaina C., Tohver E., Meert J.G., Mccausland P.J.A. \& Cocks L.R.M., 2012. Phanerozoic polar wander, paleogeography and dynamics. Earth Science Review, 114, 325-368. https://doi.org/10.1016/j.earscirev.2012.06.007.

Watycha L., 1976. Neogen niecki orawsko-nowotarskiej [The Neogene of the Orawa-Nowy Targ Basin]. Kwartalnik Geologiczny, 20, 3, 575-585.

Watycha L., 1977a. Objaśnienia do Szczegółowej mapy geologicznej Polski 1:50 000. 1047, Arkusz Jabłonka. Wydawnictwa Geologiczne, Warszawa.

Watycha L., 1977b. Szczegółowa mapa geologiczna Polski. 1047, Jabłonka. Wydawnictwa Geologiczne, Warszawa.

Wysocka A., Łoziński M., Śmigielski M., Czarniecka U. \& Bojanowski M., 2018. New data on the age of the sedimentary infill of the Orava-Nowy Targ Basin - a case study of the Bystry Stream succession (Middle/Upper Miocene, Western Carpathians). Geological Quarterly, $62,327-343$.

Zbořil L., Šefara J., Halmešová S., Král M., Pucjnerová M., Stránska M. \& Szalaiová V., 1985. Geofyzikálny výskum Turčianskej kotlina. Geofond, Bratislava [manuscript].

Zuchiewicz W., Tokarski A. K., Jarosiński M. \& Márton E., 2002. Late Miocene to present day structural development of the Polish segment of the Outer Carpathians. Stephan Mueller Special Publication Series, 3, 185-202. 